\title{
Recurrent Interactions between the Input and Output of a Songbird Cortico-Basal Ganglia Pathway Are Implicated in Vocal Sequence Variability
}

\author{
Kosuke Hamaguchi and Richard Mooney \\ Department of Neurobiology, Duke University, Durham, North Carolina, 27710
}

Complex brain functions, such as the capacity to learn and modulate vocal sequences, depend on activity propagation in highly distributed neural networks. To explore the synaptic basis of activity propagation in such networks, we made dual in vivo intracellular recordings in anesthetized zebra finches from the input (nucleus HVC, used here as a proper name) and output [lateral magnocellular nucleus of the anterior nidopallium (LMAN)] neurons of a songbird cortico-basal ganglia (BG) pathway necessary to the learning and modulation of vocal motor sequences. These recordings reveal evidence of bidirectional interactions, rather than only feedforward propagation of activity from HVC to LMAN, as had been previously supposed. A combination of dual and triple recording configurations and pharmacological manipulations was used to map out circuitry by which activity propagates from LMAN to HVC. These experiments indicate that activity travels to HVC through at least two independent ipsilateral pathways, one of which involves fast signaling through a midbrain dopaminergic cell group, reminiscent of recurrent mesocortical loops described in mammals. We then used in vivo pharmacological manipulations to establish that augmented LMAN activity is sufficient to restore high levels of sequence variability in adult birds, suggesting that recurrent interactions through highly distributed forebrain-midbrain pathways can modulate learned vocal sequences.

\section{Introduction}

Variations in vocal sequences can convey different meanings, distinguish individuals, and may also facilitate vocal learning. Consequently, neural circuitry for learned vocalizations such as speech and birdsong must modulate levels of variability for effective communication and learning. The neural mechanisms for generating variability in learned vocal sequences are poorly understood.

Birdsong comprises sequences of syllables patterned by welldefined neural circuitry, providing a system for exploring how the brain learns and controls vocal sequences (Mooney, 2009). An emerging view is that song's temporal features, including syllable sequences, are controlled by the telencephalic nucleus HVC (Vu et al., 1994; Hahnloser et al., 2002; Fee et al., 2004; Long and Fee, 2008). Interestingly, juvenile zebra finches learning to sing display elevated syllable and sequence variability (Tchernichovski et al., 2001; Olveczky et al., 2005), and these hierarchical variations are theorized to increase learning speed (Kaelbling et al., 1996; Barto and Mahadevan, 2003). Syllable variability arises

\footnotetext{
Received April 3, 2012; revised June 21, 2012; accepted July 9, 2012.

Author contributions: K.H. and R.M. designed research; K.H. and R.M. performed research; K.H. analyzed data; K.H. and R.M. wrote the paper.

This research was supported by NIH DC02524 (R.M.) and Japan Society for the Promotion of Science Postdoctoral Fellow for Research Abroad (K.H.). We thank Jennifer Baltzegar for technical assistance and Dezhe Jin, Stephen Shea, Katherine Tschida, Alex Hartemink, and Todd Roberts for critical comments on earlier versions of this manuscript. The authors declare no competing financial interests.

Correspondence should be addressed to Richard Mooney, Department of Neurobiology, Box 3209, Duke University Medical Center Durham, NC 27710. E-mail: mooney@neuro.duke.edu.

DOI:10.1523/JNEUROSCI.1666-12.2012

Copyright $\odot 2012$ the authors $\quad 0270-6474 / 12 / 3211671-17 \$ 15.00 / 0$
}

through interactions between two nuclei downstream of HVC: the output of a cortico-basal ganglia (BG) pathway LMAN and the song motor nucleus RA (robust nucleus of the arcopallium) (Kao et al., 2005; Andalman and Fee, 2009). Although inactivating LMAN during song learning reduces syllable and sequence level variability (Bottjer et al., 1984; Scharff and Nottebohm, 1991; Olveczky et al., 2005), whether augmenting LMAN activity is sufficient to elevate sequence variability remains untested and how LMAN affects sequence variability is unknown. Sequence variability could arise through LMAN-dependent modulation of HVC activity, but because LMAN axons terminate downstream of HVC, whether and how activity propagates from LMAN to HVC is unclear.

Coherency analysis of extracellular activity in LMAN and HVC of anesthetized finches reveals both HVC- and LMANleading signatures, hinting that activity propagates from LMAN to HVC (Kimpo et al., 2003; Hahnloser et al., 2006). However, these studies recorded tonically active interneurons rather than phasically bursting projection neurons (PNs) in HVC and thus may not accurately reflect the timing of activity propagating between these nuclei. Moreover, these studies did not establish a causal LMAN-HVC interaction. Another clue that LMAN can modulate HVC is that LMAN microstimulation in singing birds can trigger song truncations and restarts, a phenomenon also observed with HVC stimulation (Vu et al., 1994; Wang et al., 2008). Indeed, electrical stimulation in LMAN and the ipsilateral HVC trigger song truncations and restarts only during similar time windows in the song (Wang et al., 2008). These findings suggest that LMAN selectively modulates activity in the ipsilateral HVC, although the circuitry mediating such ipsilateral interactions remains unknown. 
Here we used dual in vivo intracellular recordings from identified HVC and LMAN PNs and pharmacological manipulations in anesthetized zebra finches to establish that activity rapidly propagates from LMAN to HVC through at least two distinct ipsilateral pathways, one of which involves a midbrain dopaminergic cell group. In vivo pharmacological manipulations were then used to establish that augmenting LMAN activity restores high levels of sequence variability in adult birds. These findings reveal that a distributed network comprising forebrain and midbrain elements underlies the songbird's capacity to modulate vocal sequence variability.

\section{Materials and Methods}

Subjects and stimuli. All experiments were carried out in accordance with a protocol approved by Duke University Institutional Animal Care and Use Committee. Data were collected from $n=80$ adult [ $>90$ posthatch days (PHDs)] male zebra finches (Taeniopygia guttata) in total: $n=22$ for dual intracellular recordings; $n=50$ for triple recordings in LMAN, HVC, and one of the afferents of HVC while stimulating LMAN electrically or chemically; $n=8$ for the behavioral (song) analysis with drug infusion through bilaterally implanted microdialysis probes.

Surgery and recording. One to five days before the experiment, birds were anesthetized with isoflurane $\left(1-2 \%\right.$ in $\left.\mathrm{O}_{2}\right)$ and placed in a stereotaxic device $\left(45^{\circ}\right.$ head angle). The scalp was dissected along the midline, and the coordinates of HVC ( $0 \mathrm{~mm}$ caudal, $2.4 \mathrm{~mm}$ lateral), LMAN (4.9 $\mathrm{mm}$ rostral, $1.85 \mathrm{~mm}$ lateral), the nucleus interface of the nidopallium (NIf; $2.25 \mathrm{~mm}$ rostral, $1.7 \mathrm{~mm}$ lateral), the medial magnocellular nucleus of the anterior nidopallium (MMAN; $5.1 \mathrm{~mm}$ rostral, $0.5 \mathrm{~mm}$ lateral), the thalamic nucleus uvaeformis (Uva; $2.25 \mathrm{~mm}$ rostral, $1.5 \mathrm{~mm}$ lateral), and A11 (1.0 mm rostral, $0.5 \mathrm{~mm}$ lateral) were determined from the caudal edge of the bifurcation of the midsagittal sinus. Local anesthetic (lidocaine cream, Fougera) was applied to the wound margins, and a stainless steel post was mounted to the rostral part of the bird's skull with dental cement.

On the morning of the electrophysiological recording, birds were injected in the pectoral muscle with $20 \%$ urethane $(80-105 \mathrm{ml}$ total; Sigma), administered in $\sim 30 \mu \mathrm{l}$ doses at $30 \mathrm{~min}$ intervals. Birds were immobilized via the mounted post in a sound-attenuating chamber (Industrial Acoustics) on an air table (Technical Manufacturing Corporation); body temperature was maintained via an electrical blanket at $37^{\circ} \mathrm{C}$ (Harvard Apparatus). Small craniotomies $(<200 \mu \mathrm{m}$ for HVC, $<400$ $\mu \mathrm{m}$ for LMAN) were made over the target nuclei, and the dura was slit open with a fine insect pin (Minuten, Carolina Biological Supply).

Sharp electrodes (borosilicate glass, BF100-50-10; Sutter Instrument) were pulled to yield a resistance of $80-150 \mathrm{M} \Omega$ when filled with $2 \mathrm{M}$ $\mathrm{K}$-acetate, 5\% neurobiotin. Hydraulic manipulators (Soma Scientific) were used to lower the electrodes into the target nuclei. For intracellular recordings, AxoClamp 2B intracellular amplifiers were used in bridge mode to measure intracellular potentials, which were low-pass filtered at $3 \mathrm{kHz}$, digitized at $11 \mathrm{kHz}$ (for some data, $10 \mathrm{kHz}$ ), and stored on a PC by using a custom software (LabView). HVC neuron types were identified on-line by their characteristic spike shape and DC-evoked firing patterns (Mooney, 2000; Mooney and Prather, 2005; Rosen and Mooney, 2006) and, in some cases, by post hoc histological methods.

For extracellular recordings, the target regions were identified by their characteristic activity patterns, including the highly correlated spontaneous bursting activity generated by HVC, NIf, and LMAN. Electrode placement in MMAN was confirmed by testing whether brief current pulses passed through the electrode could evoke orthodromic activity in HVC. For Uva, we used several criteria, including synchronized bursting activity in Uva and HVC, orthodromic activation of HVC evoked by electrically stimulating Uva, antidromic activation of Uva evoked by electrically stimulating HVC, and prolonged multiunit responses (approximately half a second) to a brief auditory stimulus (noise burst/hand clap). In fact, this prolonged response to a transient auditory stimulus was the most reliable means of locating Uva, based on post hoc histological analysis of the recording sites. In half of our recordings, we observed Uva bursts associated with the HVC bursts. In the other half, we located
Uva based on antidromic activation following HVC electrical stimulation and prolonged responses to a transient auditory stimulus. For localization of A11, we relied on stereotaxic coordinates and the presence of activity that was time-locked to bicuculline methiodide (BMI)-induced LMAN bursts.

To quantify multiunit activity from extracellular recordings, "spikes" were detected as threshold crossing events and this threshold was manually set to slightly above the floor of baseline, which was held at a fixed level across the conditions to be compared (e.g., before/after drug treatment).

For electrical stimulation experiments, bipolar electrodes were fashioned from a pair of tungsten electrodes of $100 \mathrm{k} \Omega$ resistance (Microprobes), with the tips spaced $\sim 400 \mu \mathrm{m}$. Biphasic stimulation for $400 \mu \mathrm{s}$ was applied at various current levels $(5 \mu \mathrm{A}-1 \mathrm{~mA})$.

For BMI (Sigma) injection experiments, we attached a glass pipette (tip diameter 15-20 $\mu \mathrm{m}$ ) to a tungsten electrode (0.5-1.0 M $\Omega$,). The tips of the glass pipette and the tungsten electrode were separated by $0.1-0.3$ $\mathrm{mm}$. The glass pipette was filled with $10 \mathrm{~mm}$ BMI and the BMI was injected using either a Nanoject-II (Drummond Scientific) or Picospritzer II (General Valve) in 50-200 ms pulses at 30 psi. The tungsten electrode was used to record the extracellular activity near the injection area. The glass-pipette was lowered into the target area at a $30^{\circ}$ angle through a second craniotomy to a point slightly lateral or medial $(\sim 0.6$ $\mathrm{mm}$ for LMAN, $\sim 0.25 \mathrm{~mm}$ for HVC) to the first craniotomy over the target area. BMI (10 mM) was applied until bursting activity could be detected from the intracellular and the extracellular electrodes in the target area, which in most cases required only the minimum injection volume $(4.6 \mathrm{nl})$. For GABA (250 mM) and muscimol (5 mM) injections, we used injection pipettes with similar tip diameters $(15-20 \mu \mathrm{m})$ and used a Picospritzer II to control the injections. Changes in the meniscus level in the pipette were used to calculate the injection volume.

Surgery for microdialysis probe implants. Birds were anesthetized with isoflurane (1-2\%) and placed in a stereotaxic device. Using the stereotaxic coordinates previously described, and also electrophysiological methods to localize the bursting activity that characterizes LMAN of isoflurane-anesthetized birds, microdialysis probes were implanted bilaterally into LMAN and secured in place with dental cement.

Immunohistochemical procedures. Catecholaminergic HVC projecting neurons in midbrain were identified by double labeling of retrograde fluorescent tracer injected in HVC (32 nl of 5\% Alexa Fluor 488 dextran in PBS, Invitrogen) and tyrosine hydroxylase (TH) immunoreactivity (anti-TH, Millipore Bioscience Research Reagents). A11 dopaminergic neurons are also identified as Dopamine $\beta$ hydroxylase (anti-DBH, Millipore Bioscience Research Reagents) negative cell groups.

Reversible LMAN activation/inactivation. Custom microdialysis probes were built with polyethylene tubing (Intramedic, PE-20), polyimide tubings with two different diameters (Microlumen, O.D. $=305$ and $127 \mu \mathrm{m}$ ), and the microdialysis membrane (SpectralPor, in vivo microdialysis hollow fiber, O.D. $=216 \mu \mathrm{m}$ ), based on a modified method described in (Andalman and Fee, 2009). The connections between the tubes and the end of the membrane were sealed with biocompatible epoxy (Epoxy Technology, Biocompatible Epoxy \#301). The total weight of two microdialysis probes before the implant was $<0.1 \mathrm{~g}$.

Following the implantation surgery, the reservoir was filled once a day each morning with saline until the bird began to sing consistently, typically $2-3 \mathrm{~d}$ after the implantation surgery. To infuse drugs or saline into the reservoir, the bird was placed under isoflurane anesthesia, the cap of the reservoir was removed, and the solution infused through the inlet tube until it overflowed from the outlet tube. The cap was then replaced and the bird was moved to a sound attenuation chamber to record its song activity. At the end of drug treatment days, and after recording a bird's songs for at least $8 \mathrm{~h}$, the contents of the microdialysis probe were flushed with saline following the same protocol as described for drug infusion.

MMAN lesion. First, the distribution of retrogradely labeled MMAN neurons was measured in birds that had previously $(\sim 5 \mathrm{~d})$ received fluorescent tracer injections in HVC [42-96 nl, Dextran Alexa Fluor 488 or 568 (Invitrogen)]. For these measurements, the boundaries of MMAN were defined by retrogradely labeled HVC projecting cells. The distribu- 
tion of labeled cells from the midline was measured with the Cell Counter Plugin in ImageJ, and analyzed in MATLAB. MMAN was lesioned by injecting ibotenic acid ( $2 \%$ in PBS, $54 \mathrm{nl}$ ). The extent of lesion was histologically verified from Nissl-sections, based on the microgranular appearance of the affected tissue. The percentage of MMAN lesion was obtained using the cumulative distribution of MMAN neurons as a lookup table.

Song analysis. Undirected songs were recorded from intact birds for several days before the surgery. Song was recorded using Sound Analysis Pro (SAP; http://ofer.sci.ccny.cuny.edu) and saved in wav-format files. Those sound files were analyzed by custom written software (K. Hamaguchi) in MATLAB (MathWorks). The details of song analysis procedure were as follows: sound files were digitally high-pass filtered $(>600 \mathrm{~Hz})$ and sound features, including the amplitude, entropy, frequency modulation (FM), amplitude modulation (AM), goodness of pitch, and pitch, were measured using modified program of the sound analysis for Matlab (SAM, by Sigal Saar, City College of New York, New York, NY). The sliding window size was 1024 points $(23.2 \mathrm{~ms})$. Syllables were identified as epochs where the sound amplitude remained $0.8-2$ times above the detection threshold ( 25 percentile value of the sound amplitude in a file) for $>20 \mathrm{~ms}$ and $<500 \mathrm{~ms}$. The mean and the variance of the sound features were calculated for each of the detected continuous sound periods and saved in mySQL database (ver. 4.0, http://mySQL.com).

We quantified the song activity with two measures: song continuity and sequence consistency (Scharff and Nottebohm, 1991). We defined song continuity as the median duration of continuous "chunks" of sound. The end of one continuous sound chunk was defined by silences longer than $200 \mathrm{~ms}$. Sound chunks containing only one or two sound elements were excluded from our analysis because visual inspection revealed they were almost always cage noise or other recording artifacts. $p$ values for within bird changes in song continuity on different days were assessed with a Mann-Whitney $U$-test using Bonferroni corrections as necessary.

For the syllable transition analysis, syllable types were semiautomatically identified by using a combination of manual syllable identification and a support vector machine algorithm (LIBSVM ver. 2.9 with Matlab interface, http://www.csie.ntu.edu.tw/ cjlin/libsvm). The manual and semi-automatic identification steps were repeated until there were no obvious classification errors, as determined by visual and audio inspection, to ensure that the rare syllable transition events were correctly identified. We validated the reliability of the syllable classification by using a cross-validation method. Manually classified data were randomly divided into two halves, one of which was used to train an SVM algorithm, and the other of which was used to calculate the ratio of correct classifications. All of our cross-validation values exceeded a correct classification level of $97 \%$ (i.e., $<3 \%$ error). Many of our non-dominant syllable transitions in the BMI-infused condition exceeded $p=0.03$ (see Fig. 10, transition matrix), indicating those transitions did not arise from classification error. Therefore, any changes in syllable sequences can be largely attributed to the emergence of significantly random transitions patterns and not to the misclassification of syllables. This semi-automatic classification process allowed us to identify "noise," which was eliminated from the syllable transition analysis. In the syllable transition matrix, we labeled identified syllables using lower case alphabetical characters, $\{i, a, b, c, .$.$\} , noisy unclassified syllables using an upper case N$, and the end with (End). The repetition of the same syllable was treated as a special case of syllable transition, and was not included in the syllable transition analysis. The sequence consistency is defined following the definition in the study by Scharff and Nottebohm (1991), as follows: Sequence consistency $=(\Sigma$ dominant transition per one bout $) /(\Sigma$ transition per bout). A Mann-Whitney $U$ test was used to measure the significance of the changes in song consistency and $p$ values using Bonferroni corrections as necessary.

Coherency function. To quantify the similarity of the membrane potential dynamics between HVC and LMAN neurons, spikes were first removed from the membrane potential records using a 5 ms median filter. The temporal relationship between HVC and LMAN neurons' subthreshold activities was then quantified with the coherency function. The coherency function $\gamma_{\mathrm{xy}}(t)$ measures the temporal relationship between two processes $x(t)$ and $y(t)$, which is defined as the inverse Fourier transform of the coherence $\gamma_{\mathrm{xy}}(\omega)$, as follows:

$$
\begin{gathered}
\gamma_{\mathrm{xy}}(\bar{\omega})=C_{\mathrm{xy}}(\bar{\omega}) / \sqrt{C_{\mathrm{xx}}(\bar{\omega}) C_{\mathrm{yy}}(\bar{\omega})} \\
C_{\mathrm{xy}}(\bar{\omega})=F^{-1}\left[C_{\mathrm{xy}}(t)\right] \\
C_{\mathrm{xy}}(t)=T^{-1} \int_{0}^{T} d s(x(t+s)-\bar{x}) W(t+s)(y(s)-\bar{y}) W(s),
\end{gathered}
$$

where $F[x]$ is the Fourier transform of $x$. We set $x(t)(y(t))$ as the median filtered HVC projection (LMAN) neuron's membrane potential. W $(s)$ is a symmetric Hanning window of length $T$ to remove the boundary effect. The mean membrane potential $\bar{x}$ was subtracted from the data. Equation 3 indicates that the coherency function essentially measures correlations of fluctuations around the mean (noise correlation). Note that the correlation of trial-averaged responses (signal correlation) naturally exists when two neurons have responses to a stimulus, but the existence of noise correlation or significant coherency function between indirectly connected neurons is not trivial. $T=2 \mathrm{~s}$ was the analysis window size used here. The coherency function $\gamma_{\mathrm{xy}}(t)$ is obtained by the inverse Fourier transform of $\gamma_{\mathrm{xy}}(\omega)$. We used a Gaussian window $(\mathrm{SD}=3 \mathrm{~ms})$ to smooth the coherency function. The significance of the coherency function was calculated using a $t$ test between the same-trial correlation (true data) and the trial-shuffled surrogate datasets. A significance threshold was set at $p=0.05$ with the Bonferroni correction, which corresponds to $p=1.1 \times 10^{-5}$ for each time point. For the population data, we plotted the mean and the standard deviation as the hatched region. The calculation of the coherency functions was implemented in custom written programs (K. Hamaguchi) in MATLAB (MathWorks).

Burst onset-triggered averages. When BMI was used to induce bursting activity, we used the onset of an action potential (spike) burst recorded at one recording site to trigger the average of the spike trains or medianfiltered membrane potential of the neuron at the other recording site. Spikes were detected using a threshold set typically $20-40 \mathrm{mV}$ above the baseline. The onset of a burst event was defined as the timing of the spike with a preceding interspike-interval (ISI) $>50 \mathrm{~ms}$ and with a following ISI $<5 \mathrm{~ms}$. The spike trains or median-filtered membrane potential traces were aligned to the onset of the burst events, and averaged to generate burst-triggered averages (BTAs). The significance threshold was calculated by a $t$ test between the true data (average of burst-onset triggered data) and surrogate data (random sampling of the data within the same trial, subtracted with baseline). The significance threshold was set to $p=0.01$. For the population data, the deviation from the surrogate data (natural fluctuation) was averaged. The BTA calculations were implemented in a custom written program (K. Hamaguchi) in MATLAB (MathWorks).

Synaptic onset detection. We implemented a custom synaptic onset detection algorithm based on the study by Ankri et al. (1994). Briefly, the algorithm finds events in the first derivative of voltage trace $(d V / d t)$ that exceed a threshold, which was set to 1.5 times higher than the STD of $d V / d t$ trace. An additional refractoriness parameter $(4 \mathrm{~ms})$ prevents repeated detection of the same event due to fluctuations near threshold. The algorithms described above were written in a custom MATLAB script.

Deafening. Two male zebra finches $(\sim 100$ and $\sim 110 \mathrm{dph})$ were anesthetized by Equithesin and deafened by bilateral cochlear removal. Complete removal of each cochlea was confirmed by visual inspection of the extracted cochlea under a dissecting microscope. All the behavior experiments were performed within a week after deafening.

\section{Results}

Highly correlated subthreshold activity in HVC and LMAN

The telencephalic nucleus HVC contains two distinct projection neuron types that innervate either the robust nucleus of the arcopallium $\left(\mathrm{RA} ; \mathrm{HVC}_{\mathrm{RA}}\right)$ or the striatal region Area $\mathrm{X}\left(\mathrm{HVC}_{\mathrm{X}}\right)$, both of which produce temporally sparse bursts of action potentials during 
A
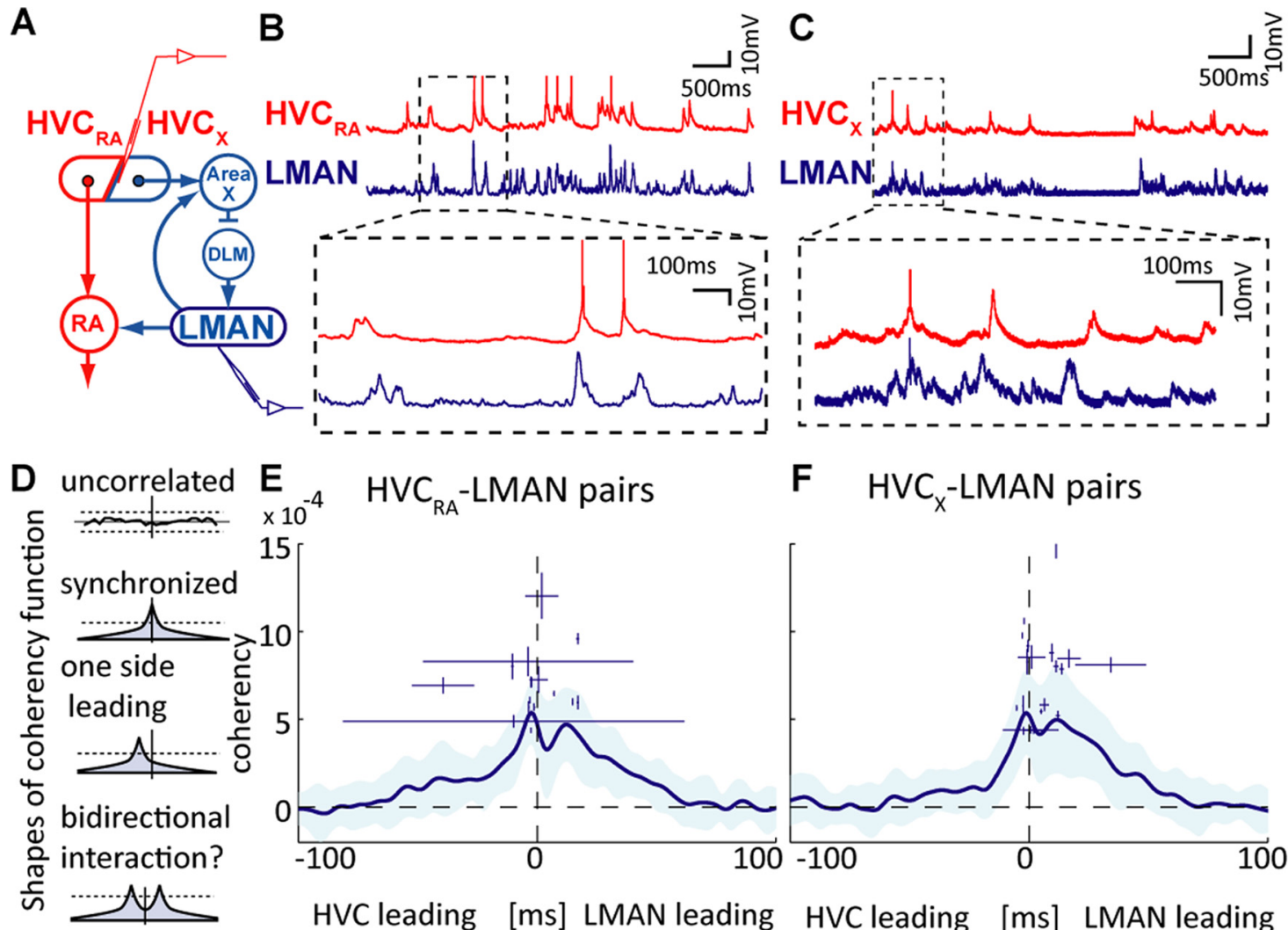

$H V C_{R A}-L M A N$ pairs

F $\quad \mathrm{HVC}_{\mathrm{x}}$-LMAN pairs
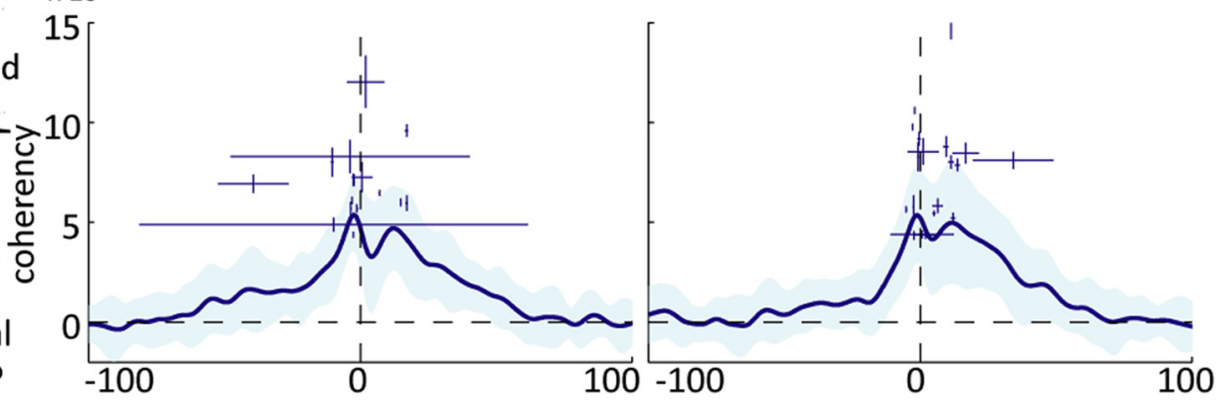

HVCleading [ms] LMAN leading

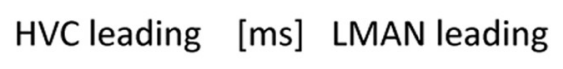

G

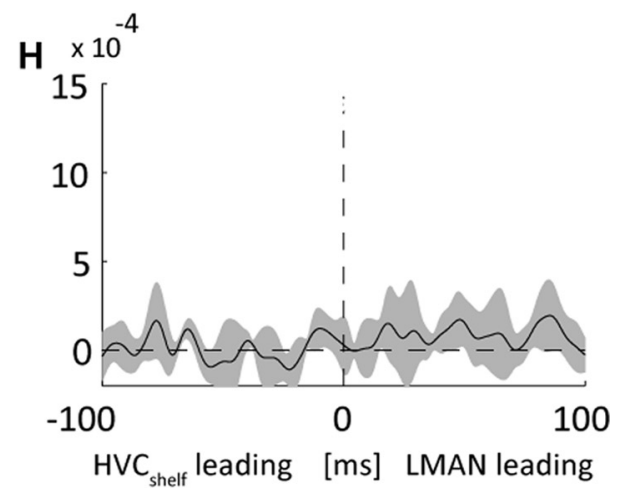

Figure 1. Correlated spontaneous activity at the input and output structures of the $B G$ pathway suggests a bidirectional interaction. $A$, Schematic of the song system showing the song motor

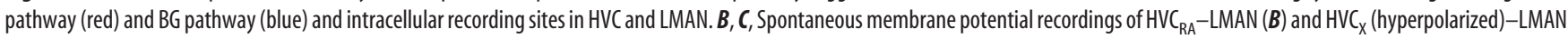
(C) neuron pairs (action potentials are clipped) show correlated synaptic activity. $\boldsymbol{D}$, Some possible shapes of coherency functions. $\boldsymbol{E}, \boldsymbol{F}$, The average coherency function of spontaneous activity recorded from $H_{V C} C_{R A}-L M A N$ pairs $(E, n=15$ of 17 pairs significant, $p<0.05)$ and $H V C_{X}-$ LMAN pairs $(\boldsymbol{F}, n=21$ of 27 pairs significant, $p<0.05)$. Shaded region, \pm 1 SD of population data. (rosses indicate the peak coherency function for individual pairs with $\pm 3 \mathrm{SD}$ of jackknife variance. $\boldsymbol{G}$, An example of an $\mathrm{HVC}_{\text {shelf }}-\mathrm{LMAN}$ paired recording. $\boldsymbol{H}$, The average coherency function of spontaneous activity recorded from $\mathrm{HVC}_{\text {shelf }}-\mathrm{LMAN}$ pairs $(\boldsymbol{E}, n=0$ of 4 pairs significant, $p>0.05)$. All $p$ values are Bonferroni-corrected.

sleep, anesthesia, and singing, and also contains interneurons, which display tonic patterns of activity in these various behavioral states (Mooney, 2000; Hahnloser et al., 2002). Earlier studies of HVCLMAN interactions predominantly sampled the activity of HVC interneurons (Kimpo et al., 2003; Rauske et al., 2003; Hahnloser et al., 2006) and thus may not accurately capture the temporal profile of activity propagating out of HVC to downstream targets, including LMAN. To more precisely examine the temporal relationship of membrane potential activity in HVC and LMAN, and to allow us to identify HVC and LMAN projection neurons through their DCevoked action potential responses (Mooney, 2000), we made sharp electrode intracellular recordings from pairs of LMAN and HVC neurons in anesthetized adult $(n=22$ birds, posthatch day $>90 \mathrm{~d})$ male zebra finches and measured spontaneous membrane potential fluctuations (Fig. 1A). To enhance our ability to detect synaptic level correlations, we injected negative current (0.1-1 nA) to suppress action potential activity and to augment depolarizing synaptic activity. As a result, our analysis was restricted to baseline membrane potential ranges between -100 and $-60 \mathrm{mV}$ for $\mathrm{HVC}_{\mathrm{RA}}$ neurons and -110 and $-80 \mathrm{mV}$ for $\mathrm{HVC}_{\mathrm{X}}$ neurons.

These recordings revealed that strongly correlated spontaneous membrane potential activity could be detected between HVC and LMAN neuron pairs (Fig. $1 B, C ; n=44$ cell pairs), regardless of whether the HVC neuron was of the type that projected to the striatal component of the $\mathrm{BG}$ pathway (i.e., $\mathrm{HVC}_{\mathrm{X}}$ cells) or to the song motor nucleus RA (i.e., $\mathrm{HVC}_{\mathrm{RA}}$ cells). In contrast, correlated membrane potential activity was not detected when paired recordings were made in the shelf region immediately ventral to 

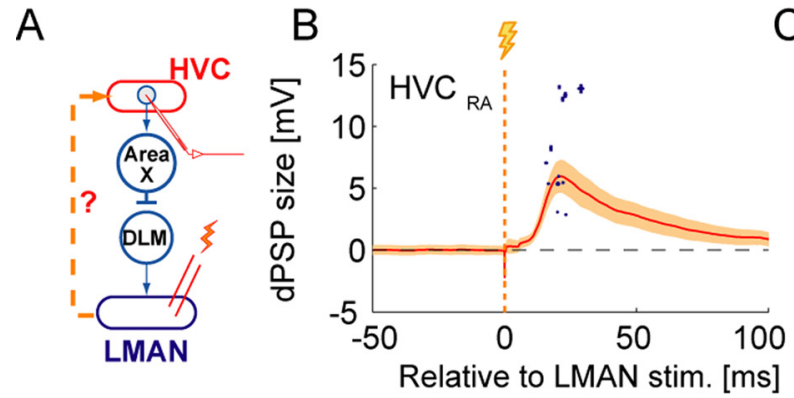

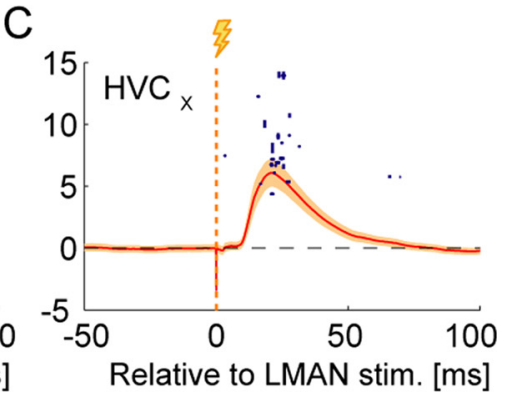

Figure 2. Electrical stimulation of LMAN induced synaptic responses in HVC. $\boldsymbol{A}$, Schematic diagram of the recording and stimulation configuration. $\boldsymbol{B}$, $\boldsymbol{C}$, Population averages of the synaptic responses evoked in $\mathrm{HVC}_{\mathrm{RA}}(\boldsymbol{B})$ and $\mathrm{HVC}_{\mathrm{X}}(\boldsymbol{C})$ neurons by brief electrical stimulation in LMAN (10-20 $\mu \mathrm{A}, 400 \mu \mathrm{s}$, bipolar electrodes). Shaded region, $\pm 1 \mathrm{SEM}$; crosses indicate the peak responses of individual cells with \pm 3 SD of jackknife variance. $\mathrm{HVC}_{\mathrm{RA}}: n=12$ of 12 cells showed significant responses; $\mathrm{HVC}_{\mathrm{x}}: n=29$ of 31 cells showed significant responses; $p<0.01$.

HVC and in LMAN (Fig. $1 G, H ; n=4$ cell pairs). This difference indicates that the strongly correlated activity we observed in HVC and LMAN is a region-specific phenomenon and did not reflect a more global synchronization of neural activity, such as up-down states observed across large extents of the cortex (Volgushev et al., 2006) or other slower state changes that might vary with the level of anesthesia or arousal.

To quantify the timing and strength of these subthreshold membrane potential correlations, we calculated a coherency function (see Materials and Methods), a form of noise correlation analysis corrected for autocorrelation effects that is especially well suited for resolving fine time scale interactions between two signals (Fig. 1D) (Kimpo et al., 2003; Hahnloser et al., 2006). Significant coherency peaks were detected in almost all HVC and LMAN pairs (36 of 44 pairs in total showed significant coherency peaks; $\mathrm{HVC}_{\mathrm{RA}}-\mathrm{LMAN}$ : 15 of 17 pairs; $\mathrm{HVC}_{\mathrm{X}}-\mathrm{LMAN}$ : 21 of 27 pairs; $p<0.05$, with Bonferroni correction). Coherency analysis revealed two interesting features of subthreshold level correlations between HVC and LMAN neurons. First, significant coherency peaks were detected for both $\mathrm{HVC}_{\mathrm{RA}}$-and $\mathrm{HVC}_{\mathrm{X}}-\mathrm{LMAN}$ pairs (Fig. 1E,F), even though both HVC cell types are separated from LMAN neurons by three or more synapses. This finding stands in contrast to the idea that significant noise correlations tend to reflect interactions between monosynaptically connected neurons (Toyama et al., 1981). Second, the peaks of the coherency function for individual pairs were bimodally distributed around $t=0$, with individual cell pairs exhibiting either an HVCleading or LMAN-leading signature (Fig. $1 E, F$ ). Such a bimodal structure is inconsistent with a circuit architecture in which HVC and LMAN receive common input, which would generate a single peak centered at $t=0$ (Fig. $1 D$ ), and is also inconsistent with a strictly feedforward interaction from HVC to LMAN (Fig. 1A) (Leblois et al., 2009), which would generate an HVC-leading peak (Fig. 1D).

\section{Evidence of bidirectional interactions between HVC and LMAN}

The failure to detect a consistent HVC-leading time signature in the coherency analysis was surprising, given the conventional view that HVC drives activity in LMAN in a feed-forward manner (Doupe et al., 2005; Roy and Mooney, 2007; Leblois et al., 2009). One possibility is that the bimodal structure of the average coherency function we observed reflects reciprocal interactions between HVC and LMAN. The circuit basis for such a reciprocal interaction has not been described. To begin to explore the circuit basis for such an interaction, we electrically stimulated LMAN (10-20 $\mu \mathrm{A}, 400 \mu \mathrm{s}$, bipolar electrodes) and recorded membrane potential activity in HVC projection neurons using sharp intra- cellular electrodes (Fig. 2A). To maximize our ability to detect synaptic responses in HVC, we maintained the impaled cell's resting membrane potential $<-80 \mathrm{mV}$, using tonic hyperpolarizing current, as needed. Interestingly, LMAN stimulation evoked depolarizing synaptic responses in almost all of the HVC projection neurons we examined (Fig. $2 \mathrm{~B}, \mathrm{C} ; 12$ of $12 \mathrm{HVC}_{\mathrm{RA}}$ neurons and 29 of $31 \mathrm{HVC}_{\mathrm{X}}$ neurons showed significant responses to LMAN stimulation; $t$ test, threshold $p=0.01$ ). The average latency of evoked synaptic onset responses was $\sim 13 \mathrm{~ms}$ $\left[\mathrm{HVC}_{\mathrm{RA}}: 13.4 \pm 0.2 \mathrm{~ms}\right.$ (median \pm Jackknife $\mathrm{SD}$, quartile range: $11.2-14.5 \mathrm{~ms}$ ); $\mathrm{HVC}_{\mathrm{X}}$ : $13.0 \pm 0.05 \mathrm{~ms}$ (quartile range: $11.8-15.4$ $\mathrm{ms})$ ], consistent with the idea that LMAN stimulation excites HVC neurons via a multisynaptic pathway.

One potential confound is that the axons of some $\mathrm{HVC}_{\mathrm{X}}$ neurons course through LMAN en route to the striatum, raising the possibility that electrical stimulation in LMAN could antidromically activate $\mathrm{HVC}_{\mathrm{X}}$ neurons, which in turn could excite other HVC neurons through their local axon collaterals (Mooney and Prather, 2005). To avoid this potential confound, we induced synchronized, rhythmic bursting activity in LMAN by local application of BMI (10 mM) and made intracellular recordings from pairs of $\mathrm{HVC}_{\mathrm{X}}$ and LMAN neurons in anesthetized finches. When BMI was applied to LMAN, action potential bursts in LMAN were closely followed by synaptic activity in simultaneously recorded $\mathrm{HVC}_{\mathrm{X}}$ neurons (Fig. $3 A$ ). Averaging the $\mathrm{HVC}_{\mathrm{X}}$ membrane potential relative to the first action potential of the LMAN neuronal burst revealed a depolarizing response in HVC with a peak latency of $\sim 20 \mathrm{~ms}$ [Fig. $3 B$; peak $=21.3 \pm 2.9 \mathrm{~ms}$ (mean \pm SEM), $n=7$ of 7 pairs showed significant peaks]. Coherency analysis of LMAN-HVC neuronal pairs recorded when BMI was applied in LMAN revealed a single peak with an LMANleading signature (Fig. 3E, blue line). To further explore the reciprocal nature of HVC-LMAN interactions, we used BMI to induce synchronized bursting activity in HVC. Under these conditions, action potential bursts in HVC were followed by a depolarizing response in LMAN (Fig. 3C,D; mean peak $=16.7 \pm 5.7$ $\mathrm{ms}, n=8$ of 8 pairs showed significant peaks) and the coherency analysis revealed a single HVC-leading peak (Fig. 3E, red line). Thus, synchronizing the bursting activity of neurons in either HVC or LMAN shifts the coherency peak in opposite directions, consistent with a reciprocally connected network.

\section{Activity propagates from LMAN to HVC through Area X but not through robust nucleus of the arcopallium or dorsal arcopallium}

The present finding that relatively long-latency (15-20 ms) synaptic responses could be readily evoked in HVC by electrical or chemical stimulation of LMAN is consistent with the idea that 
A
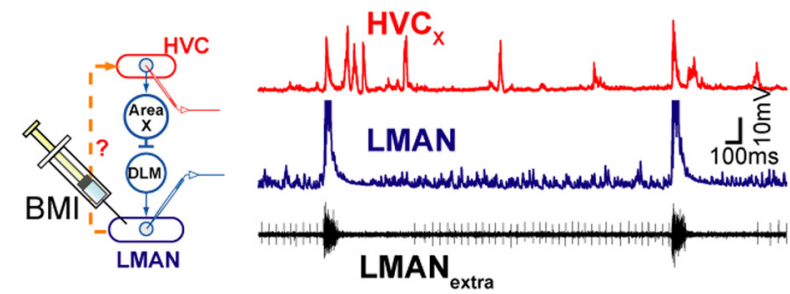

C
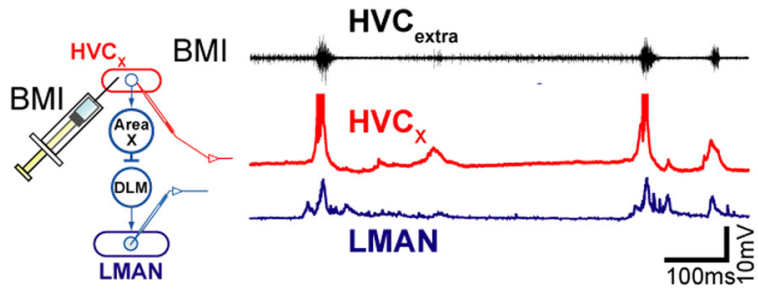

E
B

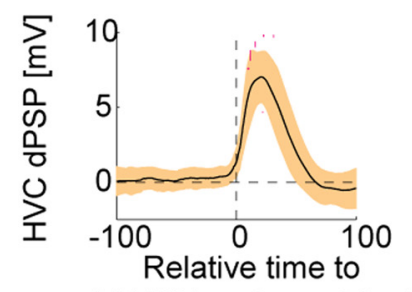

LMAN burst onset [ms]
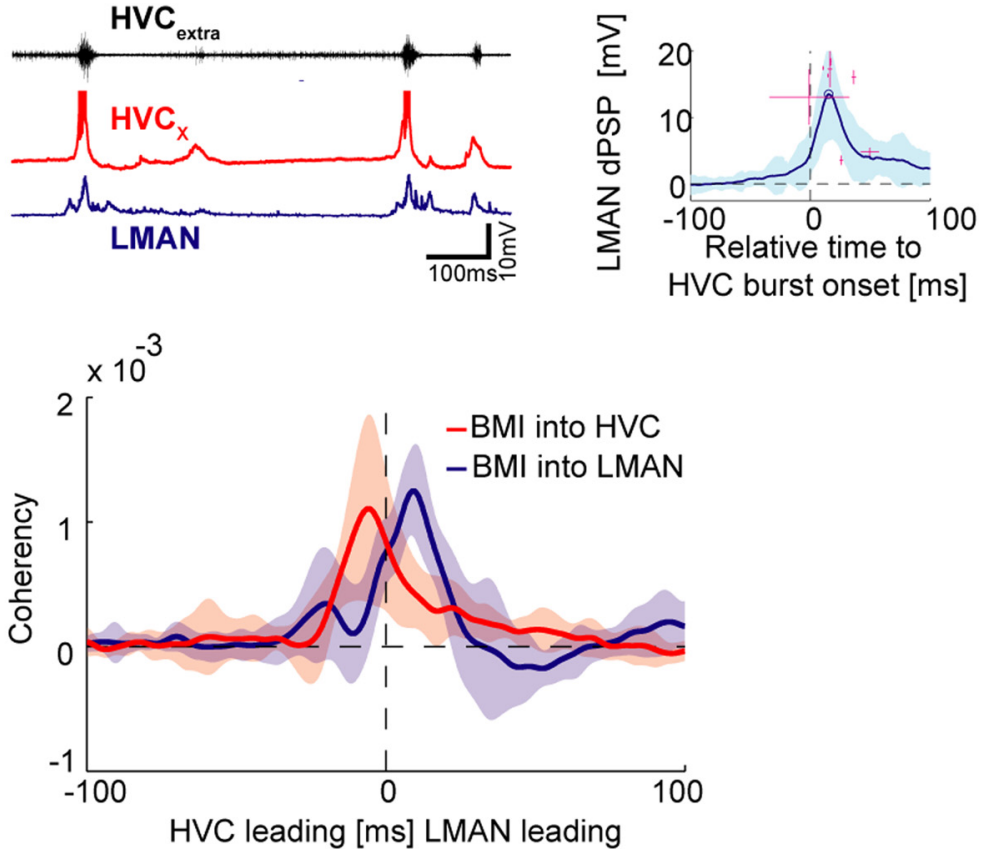

Figure 3. Local pharmacological manipulation of activity provides evidence of bidirectional interactions between HVC and LMAN. $A$, In vivo intracellular recording of an HVC ${ }_{x}$ (red) and LMAN (blue) neuron pair and extracellular recording in LMAN (black) after BMI (10 mM) injection into LMAN. $\boldsymbol{B}$, Average HVC $C_{x}$ neuron voltage aligned to BMI-induced action potential burst onset in LMAN shows an $\mathrm{HVC}_{\mathrm{X}}$-following peak ( $t=21.3 \pm 2.9 \mathrm{~ms}, n=7$ pairs). Shaded region, $\pm 1 \mathrm{SD}$; crosses indicate the peak responses for individual cells with jackknife variance; square with error bar indicates median with quartile. C, In vivo intracellular recording of an $\mathrm{HVC}_{x}$ (red) and LMAN (blue) neuron pair and extracellular recording in HVC (black) after BMl injection into HVC. D, Average LMAN

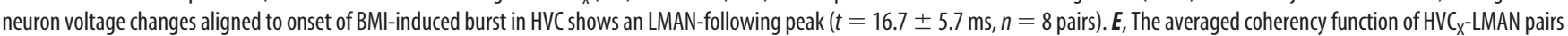
when BMI was applied to either HVC (red, $n=8$ pairs) or LMAN (blue, $n=7$ pairs).

these two structures are linked by a recurrent multisynaptic circuit. Although the architecture of this circuit is unknown, it must depend on either or both of the efferent targets of LMAN, Area X and RA. To begin to investigate how activity propagates from LMAN to HVC, we first worked forward from LMAN, by exploring whether inactivating either Area X or RA could block propagation of activity from LMAN to HVC. We made simultaneous extracellular multiunit recordings from HVC, LMAN, and either Area X or RA; the electrode placed in either of these latter structures also included a piggyback puffer pipette containing lidocaine (4\% in PBS). Local application of BMI (10 mM; 4.6-32 nl, Nanoject II; see Material and Methods) was then used to induce synchronized, rhythmic bursting activity in LMAN. We then calculated LMAN burst-onset triggered PSTHs of multiunit activity (MUA) recorded in HVC for 5 min before and immediately after applying lidocaine $(40-80 \mathrm{nl})$ to either Area X or RA. These experiments revealed that Area $\mathrm{X}$ inactivation strongly suppresses the HVC MUA responses that follow LMAN bursts $(n=$ 5 , paired $t$ test of HVC peak responses, $p<0.001$; Fig. $4 B$ ). In contrast, lidocaine injection in RA did not affect HVC responses to LMAN bursting activity $(n=4$, paired $t$ test, $p>0.5$; Fig. $4 C, D)$. The finding that RA inactivation failed to block HVC responses was surprising because high intensity electrical stimulation in RA is known to activate HVC bilaterally through a recurrent brainstem pathway (see detailed pathway in Fig. $6 \mathrm{~A}$ ) that involves Uva (Wild, 2004; Ashmore et al., 2008). Furthermore, a subset of neurons in the dorsal portion of RA projects to the ipsilateral HVC (Roberts et al., 2008). Nonetheless, these findings indicate that activity propagates from LMAN to HVC through Area $\mathrm{X}$ and not through RA.

Although we were careful to target LMAN precisely and use small injection volumes of BMI ( $\sim 4.6 \mathrm{nl})$, experiments using local application of BMI or weak electrical stimulation in LMAN cannot avoid affecting regions immediately surrounding this nucleus. This is potentially problematic, because the region immediately surrounding LMAN ( $\left.\mathrm{LMAN}_{\text {shell }}\right)$ is speculated to communicate with HVC through a polysynaptic pathway that involves the dorsal arcopallium [Ad; i.e., $\mathrm{LMAN}_{\text {shell }}-\mathrm{Ad}$-dorsal thalamic zone-MMAN-HVC (Johnson et al., 1995; Bottjer and Altenau, 2010)] Therefore, one possibility is that activation of the 


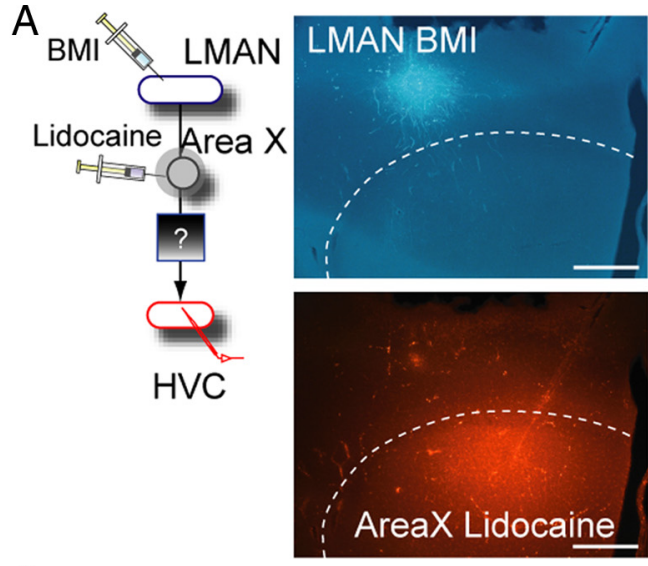

C

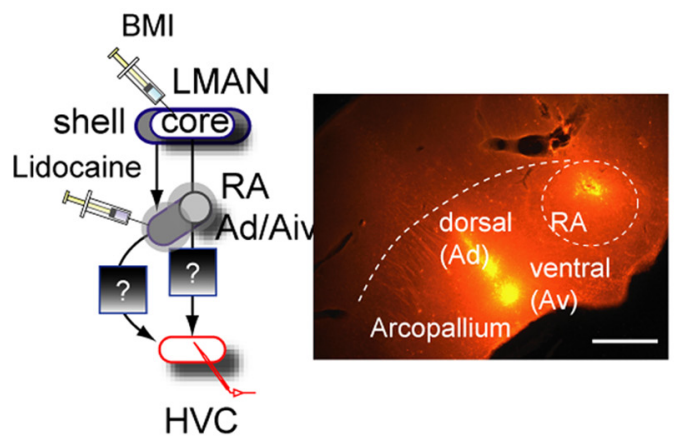

$\mathrm{E}$

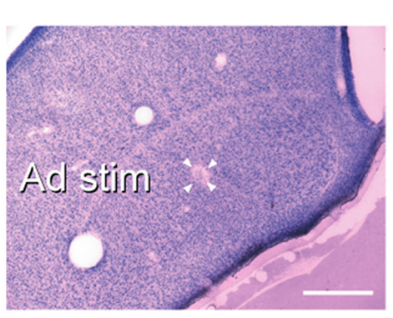

HVC MUA

RA stim

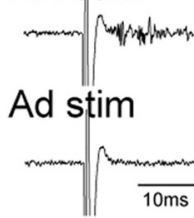

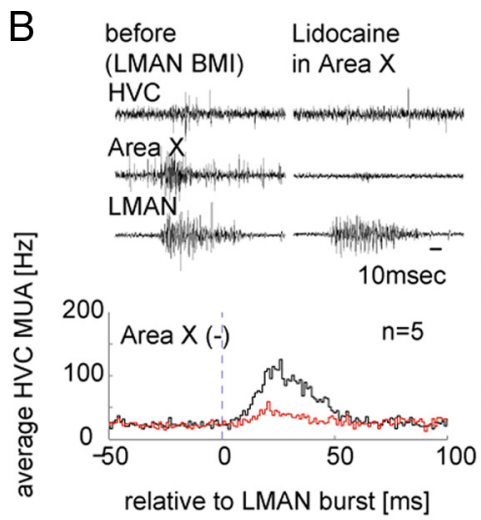
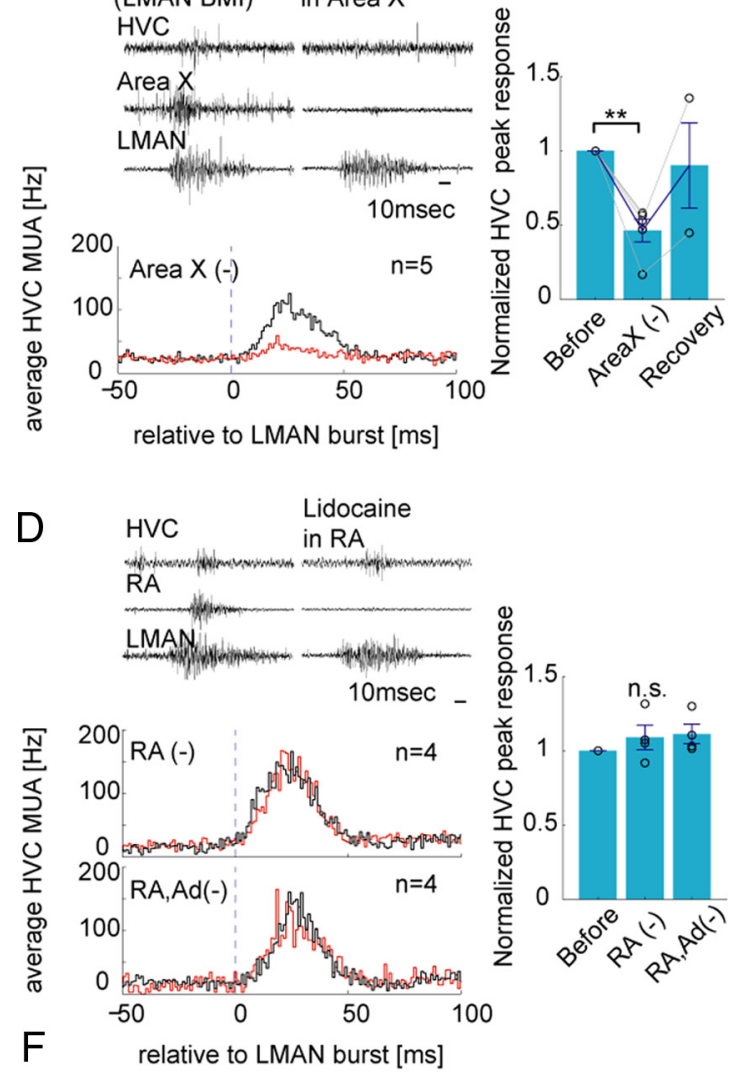

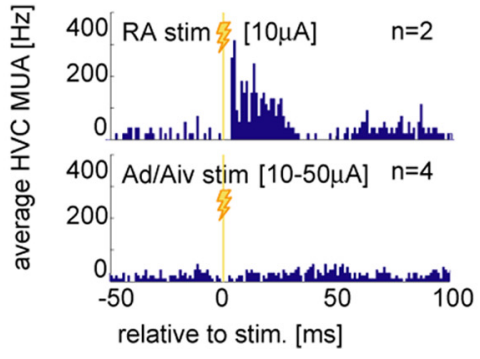

Figure 4. HVC responses to LMAN bursting activity are blocked by Area X inactivation, but not by inactivation of RA and Ad. A, Left, Schematic diagram of the stimulation, recording and inactivation pipettes. Right, An example of LMAN BMI (top) and Area X Lidocaine (bottom) injections visualized by dextran fluorescent dyes mixed in the drug solution [LMAN; dextran Alexa Fluor 488 (Invitrogen), Area X, dextran Texas Red (Invitrogen)]. B, Top left, Traces of MUA recorded in HVC, Area X, and LMAN before and after Lidocaine injection (4\%, $40-80 \mathrm{nl}$ ) in Area X. Bottom left, Averaged HVC MUA response to LMAN burst onset before (solid) and after (red) Area X inactivation. Right, Normalized HVC peak MUA responses before Area Xinactivation, during Area X inactivation $($ Area X $(-)$ ), and upon recovery $(\sim 2 \mathrm{~h})$. C, Left, Schematic diagram of the stimulation and recording configuration used in RA/Ad inactivation experiments. Right, An example of Lidocaine injection sites in RA and Ad/Av visualized by dextran Texas Red mixed in the Lidocaine solution. D, Top left, Traces of MUA recorded in HVC, RA, and LMAN before and after Lidocaine injection (4\%, 40 - $80 \mathrm{nl}$ ) in RA. Middle and bottom left, Averaged HVC MUA response to LMAN burst onset before (solid) and after (red) RA inactivation and before and after Ad/Av inactivation (bottom). Right, Normalized HVC peak MUA responses before RA inactivation, during RA inactivation ( $\mathrm{RA}(-))$, and during both $\mathrm{RA}$ and $\mathrm{Ad} / \mathrm{Av}$ inactivation $(\mathrm{RA}, \mathrm{Ad}(-))$. $\boldsymbol{E}$, Left, Histological verification of a stimulation site in $\mathrm{Ad}$. Right, Example traces of HVC extracellular recording with electrical stimulation in RA or Ad (both $10 \mu \mathrm{A})$. F, Average HVC MUA response to RA stimulation ( $n=2$ stimulation sites from two birds) and Ad/Av stimulation ( $n=4$ stimulation sites from two birds). ${ }^{* *} p<0.01$.

$\mathrm{LMAN}_{\text {shell}}$-Ad pathway may account for the presumed propagation of activity from LMAN to HVC.

To test this possibility, we further explored whether activity in Ad was necessary for the HVC responses that followed LMAN bursting activity. Following inactivation of RA as described above, we stereotaxically injected lidocaine $(4 \%, \sim 150 \mathrm{nl})$ at multiple depths $(2200-3300 \mu \mathrm{m})$ to inactivate dorsal and ventral arcopallium (Ad/Av; Fig. 4C). Inactivation of this large extent of $\mathrm{Ad} / \mathrm{Av}$ in addition to inactivation of RA did not affect the HVC responses that followed bursts of activity in LMAN ( $n=4$, paired $t$ test, $p>0.5$; Fig. $4 D$ ). These data further strengthen a model in which LMAN activity propagates through Area X to reach HVC, and diminish support for the idea that the LMAN $_{\text {shell }}-$ Ad pathway influences HVC activity in an acute manner.

To further evaluate the potential of the LMAN $_{\text {shell }}-$ Ad pathway to signal HVC, we stimulated Ad/Av with a monopolar electrode while recording multiunit activity in $\mathrm{HVC}(10-50 \mu \mathrm{A}$ current intensity; single pulse of $400 \mu \mathrm{s})$. Electrical stimulation in Ad/Av did not drive HVC activity (Fig. $4 E, F ; n=4$ stimulation sites from two birds), even though much lower currents applied in RA readily drove multiunit responses in HVC (Fig. 4E, F; 10 $\mu \mathrm{A}, n=2$ stimulation sites from two birds). These results suggest that the LMAN $_{\text {shell}}$-Ad pathway does not communicate with HVC, as previously speculated (Bottjer and Altenau, 2010). 
A

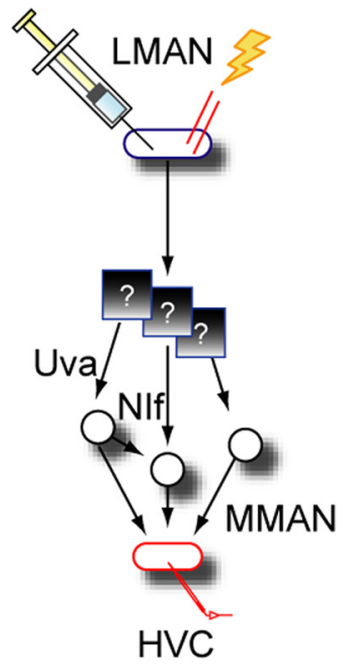

D

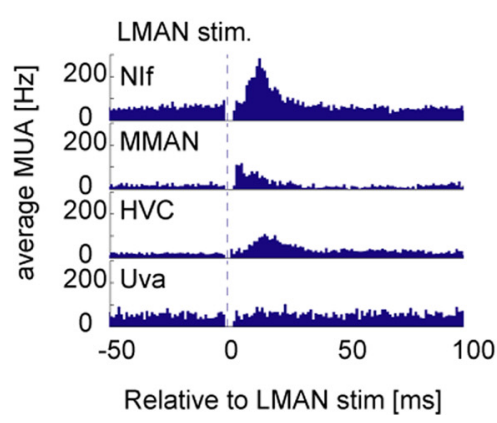

LMAN stim

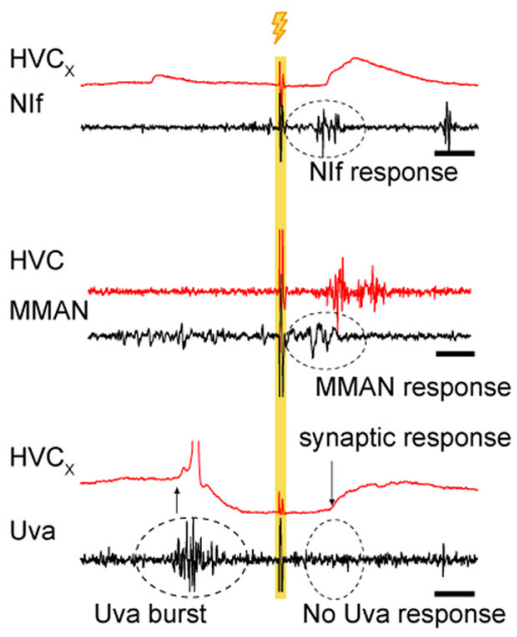

E

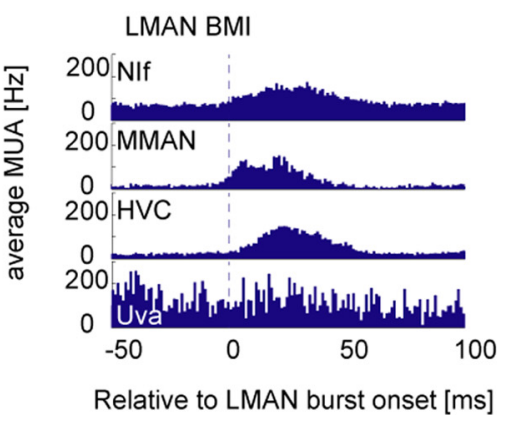

C

BMI induced LMAN burst
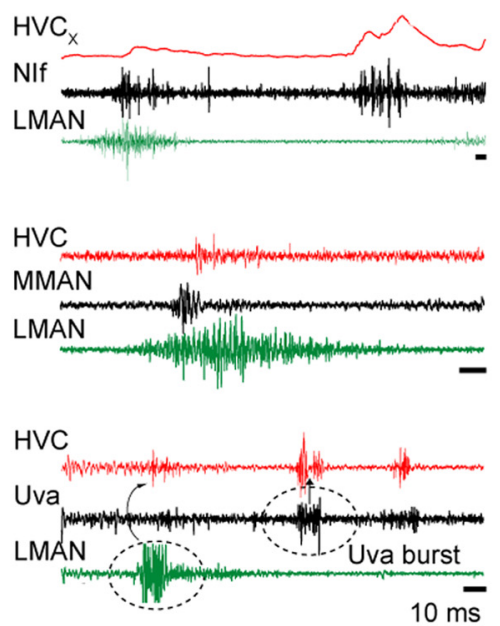

F

Nif

LMAN stim.

MMAN

HVC

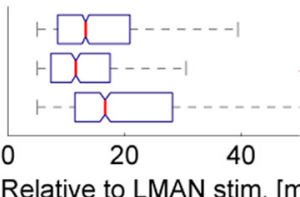

Relative to LMAN stim. [ms]

LMAN BMI.

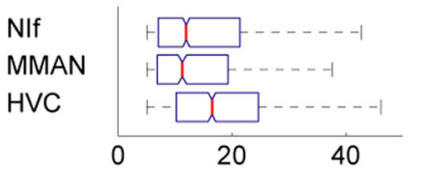

Relative to LMAN burst onset [ms]

Figure 5. Electrical or chemical stimulation of LMAN drives responses in NIf and MMAN, but not in Uva. $\boldsymbol{A}$, Schematic diagram of the stimulation and recording configuration. $\boldsymbol{B}$, Traces of MUA extracellularly recorded in NIf, MMAN, and Uva before and after electrical stimulation in LMAN (10-20 $\mu$ A; marked by arrow).C, Extracellular or intracellular recordings of activity in NIf, MMAN, Uva, and HVC following chemically-induced bursting activity in LMAN (BMI, $10 \mathrm{~mm} ; 4.6-32 \mathrm{nl} ; 100-197 \mu \mathrm{m}$ radius sphere). $\boldsymbol{D}, \boldsymbol{E}$, Population averages of the MUA histograms of NIf, MMAN, HVC, and Uva activity aligned to electrical stimulation (D) or the onset of BMI-induced bursting $(\boldsymbol{E})$ in LMAN. Significant responses to either form of LMAN stimulation were detected in NIf, MMAN, and HVC, but not in Uva. $\boldsymbol{F}$, Latency plots of multiunit extracellular activity evoked in NIf, MMAN, and HVC, showing median, first, and third quartiles.

\section{Identifying circuitry that links LMAN to HVC}

To further explore how activity propagates from LMAN to HVC, we also mapped the intervening functional circuitry in a backwards direction from HVC. The most proximal node of this circuitry must include one or more of the afferents of HVC, the most prominently studied of which include three forebrain nuclei: MMAN, NIf, and Uva. To further investigate how activity propagates from LMAN to HVC, we made intracellular or extracellular recordings from $\mathrm{HVC}$ and extracellular recordings from one or more of these afferent nuclei while stimulating LMAN either electrically or chemically (i.e., with BMI) (Fig. $5 \mathrm{~A}$ ).

Brief electrical stimulation in LMAN $(10-20 \mu \mathrm{A} ; 400 \mu$ s, bipolar electrodes) reliably evoked MUA responses in NIf and MMAN and synaptic and/or MUA responses in HVC but failed to evoke any responses in Uva (Fig. $5 B$; NIf: $n=12$ recordings sites in 12 birds had significant responses, $p<0.0008$; MMAN: $n=5$ sites in 5 birds had significant responses, $p<0.017$; HVC: $n=12$ sites in 12 birds had significant synaptic or MUA responses, $p<0.004$; Uva: $n=0$ sites in 3 birds had significant responses, threshold $p=0.05)$. An estimation of response latencies based on the first "spike" in the evoked MUA revealed that responses in MMAN and NIf preceded MUA response onset in HVC by 3-5 ms, consistent with a monosynaptic connection $[\mathrm{MMAN}$ : latency $=11.7 \pm 0.04 \mathrm{~ms}($ median \pm
Jackknife SD, quartile range: 7.35-17.5 ms); NIf: latency $=13.3 \pm$ $0.01 \mathrm{~ms}$ (quartile range: $8.53-21.0 \mathrm{~ms}$ ); HVC: latency $=16.7 \pm 0.01$ ms (quartile range: $11.5-28.2 \mathrm{~ms}$ )]. Although LMAN stimulation did not evoke responses in Uva, the simultaneous recordings made in Uva and HVC often detected characteristic bursting activity in Uva that preceded action potential responses in $\mathrm{HVC}_{\mathrm{X}}$ cells, indicating that LMAN and Uva influence HVC through independent channels (Fig. 5B).

We also used chemical activation of LMAN to map the circuitry by which information flows to HVC, thus avoiding the fibers of passage confound that electrical stimulation may potentially create. Using the strategy previously described, we injected small volumes of BMI (10 mM; 4.6-32 nl) at the lateral edge of LMAN. We then calculated LMAN burst-onset triggered PSTHs of MUA recorded in NIf, MMAN, Uva, and HVC to measure response strength and latency. This analysis revealed the same pattern of interactions as did electrical stimulation: BMI-induced bursting activity in LMAN correlated with reliable following activity in NIf, MMAN, and HVC, but not in Uva (Fig. 5C,E; NIf: $n=11$ sites in 11 birds showed significant responses, $p<0.006$; MMAN: $n=5$ sites in 5 birds showed significant responses, $p<$ $0.081 \times 10^{-9}$; HVC; $n=16$ sites in 16 birds showed significant responses, $p<0.001$; Uva: $n=0$ sites in 2 birds had significant 
A

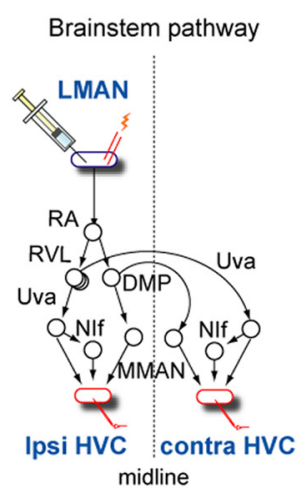

B
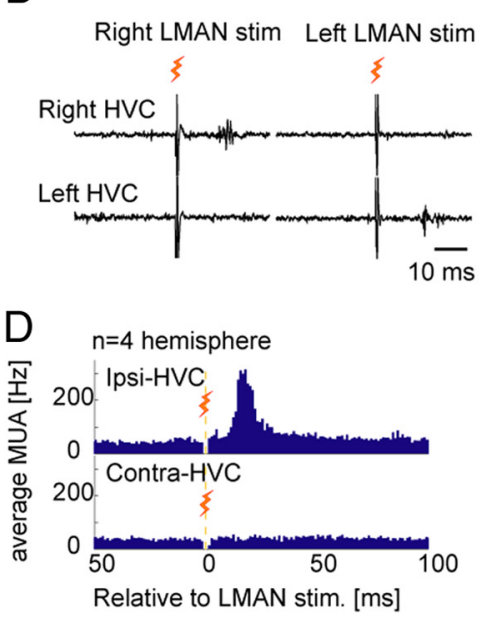

C

Right LMAN BMI

Left HVC
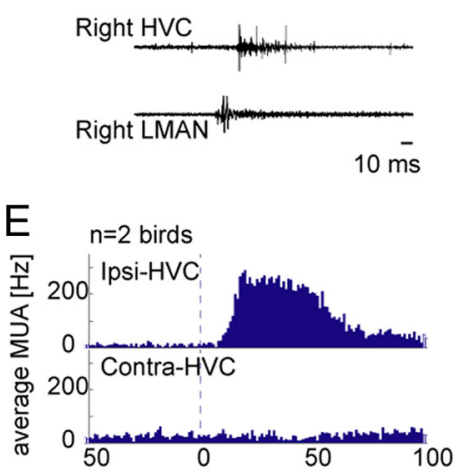

Relative to LMAN burst onset [ms]

Figure 6. Unilateral electrical or chemical (BMI) stimulation of LMAN evokes responses in the ipsilateral but not contralateral HVC. A, Schematic diagram of the stimulation and recording configuration, including the recurrent brainstem pathway that is one possible pathway that may link LMAN to HVC. $\boldsymbol{B}$, An example of the lateralized response evoked in HVC by electrical stimulation in LMAN. Bilateral HVC recordings made in the same bird reveal that unilateral LMAN stimulation evokes responses only in the ipsilateral HVC. C, An example of the lateralized response evoked in HVC by BMI-induced bursting activity in LMAN. D, Population average of simultaneously recorded ipsilateral and contralateral HVC MUA aligned to LMAN stimulation (red lightning bolt): all ipsilateral HVC recordings showed significant responses ( $n=4$ hemispheres, $p<3 . \times 10^{-18}$ with Bonferroni), whereas no contralateral HVC recordings showed significant responses ( $n=4$ hemispheres, $p>0.01$ ). $E$, Population average of HVC MUA aligned to the onset (dashed vertical line) of bursting activity induced in LMAN by focal BMl injection. All ipsilateral HVC recordings showed significant responses ( $n=2$ birds, $p<1.8 \times 10^{-21}$ with Bonferroni) following the LMAN burst onset, whereas no contralateral HVC recordings showed significant responses ( $n=2$ birds, $\left.p>0.01\right)$. RVL, Rostral ventrolateral medulla; DMP, dorsomedial nucleus of posterior thalamus.

responses to LMAN bursting, threshold $p=0.05)$. Although the population average of LMAN burst-onset triggered responses showed longer and broader responses compared with responses evoked by LMAN electrical stimulation, the dominant pattern was always LMAN leading (Fig. 5E), with latencies that were highly similar to those obtained with electrical stimulation [Fig. 5F; MMAN: latency $=11.3 \pm 0.11 \mathrm{~ms}$ (median \pm Jackknife SD, quartile range: $6.89-19.3 \mathrm{~ms}$ ); NIf: latency $=12.0 \pm 3.2 \times 10^{-13}$ ms (quartile range: 7.07-21.4 ms); HVC: latency $=16.5 \pm 1 \times$ $10^{-13}$ (quartile range: $10.2-24.7 \mathrm{~ms}$ )]. Along with the electrical stimulation experiments, these findings support the idea that LMAN activity can access HVC through at least two different pathways that do not involve the thalamic nucleus Uva.

The finding that LMAN stimulation failed to drive activity in Uva is consistent with the idea that LMAN activity completely bypasses RA and the downstream, recurrent brainstem pathway, the ascending portions of which make bilateral projections to Uva and thus HVC (Wild, 2004; Ashmore et al., 2008). This led us to explore whether the circuitry underlying the LMAN-HVC interactions we observed involves unilateral or bilateral architecture. To resolve this issue, we recorded multiunit activity in the ipsilateral and contralateral HVC while electrically or chemically stimulating LMAN (Fig. 6A). Electrical stimulation routinely evoked responses in the ipsilateral $\mathrm{HVC}$, but failed to evoke any responses in the contralateral HVC (Fig. $6 B$; ipsilateral HVC: $n=$ 4 of 4 hemispheres showed significant responses, $p<3$. $\times 10^{-18}$; contralateral HVC: $n=0$ of 4 hemisphere showed significant responses, threshold $p=0.01$, tested up to $1 \mathrm{~mA}$ ). Qualitatively similar results were obtained with chemical stimulation (Fig. 6E; ipsilateral HVC: $n=2$ of 2 hemispheres showed significant responses, $p<1.8 \times 10^{-21}$; contralateral HVC: $n=0$ of 2 hemispheres showed significant responses, threshold $p=0.01)$. These experiments reveal that LMAN activation drives only the ipsilateral HVC, providing further evidence that the circuitry that conveys information from LMAN to HVC is separate from recurrent forebrain-brainstem circuitry, the ascending portions of which make bilateral projections to HVC.
LMAN communicates with HVC through a pathway that does not depend on NIf or MMAN

The current findings that LMAN activity drives both NIf and MMAN suggest two possible conduits for linking LMAN to HVC. To directly test whether these structures were necessary for activity to propagate from LMAN to HVC, we electrically or chemically stimulated LMAN while making electrophysiological recordings in HVC and reversibly inactivating NIf and/or MMAN. Inactivating NIf with concentrated (250 mM) GABA actually augmented the HVC response evoked by electrical stimulation or BMI-induced bursting in LMAN, even though this treatment strongly suppressed spontaneous activity in HVC, as previously reported (Fig. $7 B-D$; electrical stimulation: $n=5$ neurons from 5 birds; BMI: $n=3$ cells from 3 birds; $p<0.0001$ ) (Coleman and Mooney, 2004; Bauer et al., 2008). A similar set of experiments using extracellular recordings in HVC and muscimol to sequentially block activity in MMAN and NIf also failed to disrupt the propagation of BMI-evoked activity from LMAN to HVC (Fig. 7E-G). Thus, LMAN activates HVC through an ipsilateral pathway that does not depend solely on any of the forebrain afferents of HVC.

A midbrain dopaminergic cell group links LMAN to HVC A remaining possibility is that LMAN signals HVC through structures that reside below the level of the forebrain. In fact, neuroanatomical studies in canaries have established that HVC receives input from neuromodulatory centers in the midbrain, including the dopaminergic cell group A11 (Appeltants et al., 2000). Although a traditional view is that the neuromodulatory nature of this cell group could not account for the rapid propagation of activity from LMAN to HVC, we explored whether A11 functions as an intermediary between LMAN and HVC. First, using a combination of retrograde tracers injected into HVC and immunohistochemical methods to identify neurons positive for the catecholaminergic marker $\mathrm{TH}$, we confirmed that $\mathrm{TH}^{+}$neurons in A11 provide monosynaptic input to HVC (Fig. 8 A). Most $\mathrm{TH}^{+}$HVC-projecting A11 neurons were located near the mid- 
A

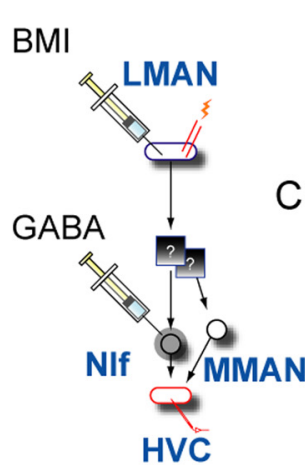

E

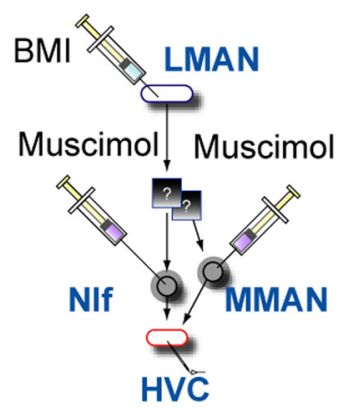

B
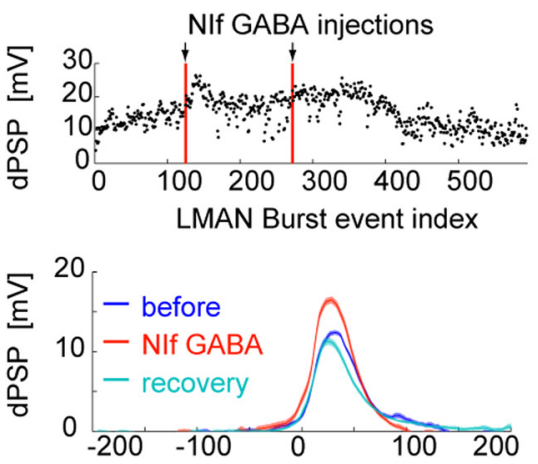

Relative time to LMAN burst onset [ms]

$\mathrm{F}$

HVC multiunit response to LMAN BMI

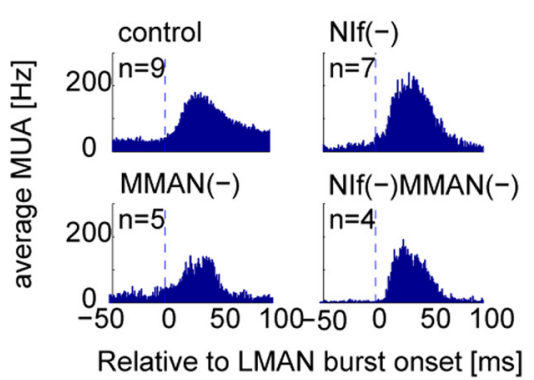

D

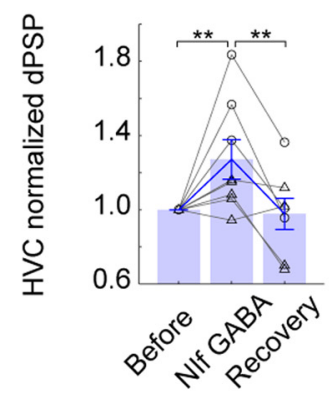

G

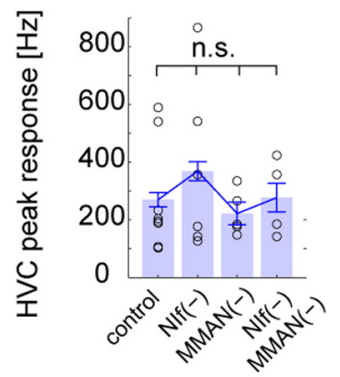

Figure 7. Inactivating NIf and/or MMAN fails to block propagation of BMI-induced bursting activity from LMAN to HVC. $A, A$ schematic of the stimulation, inactivation, and recording configuration. $\boldsymbol{B}$, The peak amplitude of depolarizing postsynaptic potentials following each LMAN burst are plotted before, during, and after NIf inactivation [NIf GABA (250 mM)]. C, LMAN burst-triggered averaged dPSP responses in HVC before (blue), during [NIf GABA (red)], and after (recovery; cyan) Nlf inactivation (traces are plotted with SEM, using the same data from $\boldsymbol{A}$ ). $\boldsymbol{D}$, Population average of the normalized dPSP distribution before, during, and after NIf inactivation. For this plot, both LMAN electrical stimulation cases ( $n=5$ birds, triangles) and LMAN BMI cases ( $n=3$ birds, circles) are pooled together. NIf inactivation augmented dPSP amplitude of HVC projection neurons $[n=4$ out of 5 birds in LMAN stimulation, $n=3$ out of 3 birds in LMAN BMI, significant increases during NIf inactivation $(p<0.0001)$ ]. $\boldsymbol{E}$, Schematic diagram of the experiment. $\boldsymbol{F}$, Average HVCMUA aligned to the onset of BMI-induced bursts in LMAN in four conditions; control, when NIf was inactivated with muscimol (5 mM) (NIf(-)), when MMAN was inactivated with muscimol (MMAN(-)), and when NIf and MMAN were inactivated with muscimol (NIf( $(-)$ MMAN $(-))$. Inactivating NIf and MMAN did not stop the propagation of activity from LMAN to HVC. G, Peak responses of HVC MUA aligned to LMAN burst onset did not show significant differences under the four conditions (ANOVA, $p=0.66){ }^{* *} p<0.01$.

line $(0-700 \mu \mathrm{m})$ and an additional population of $\mathrm{TH}^{-} \mathrm{HVC}-$ projecting neurons was more widely distributed over the lateral part of the central gray region (GCt). Furthermore, the A11 cell group did not show positive staining for dopamine $\beta$ hydroxylase (data not shown), the synthetic enzyme that is a marker of noradrenergic and adrenergic neurons, supporting the conclusion that $\mathrm{TH}^{+}$A11 cells are dopaminergic. Thus, similar to the anatomical organization in the canary, HVC in the zebra finch receives input from a midbrain cell group that includes a midline population of dopaminergic neurons.

To determine whether A11 could link LMAN to HVC, we made simultaneous extracellular recordings from $\mathrm{HVC}, \mathrm{A} 11$, and LMAN and used focal injections of BMI ( $10 \mathrm{~mm}, 4.6 \mathrm{nl})$ to induce bursting activity in LMAN (Fig. $8 B$ ). These recordings revealed that bursts of A11 neuronal activity followed the onset of bursting activity in LMAN and preceded the onset of the LMAN-evoked response in HVC (Fig. 8B,C). Moreover, A11 activity reliably followed the onset of LMAN bursting activity in all birds that we examined ( $n=5$ sites in 5 birds showed significant responses, $p<$ 0.00006), consistent with the idea that LMAN activity drives this midbrain cell group. A remaining issue is whether A11 can drive HVC at the short latencies necessary to account for the relatively rapid propagation of activity from LMAN to HVC, especially given the classical view that midbrain dopaminergic neurons primarily function to slowly modulate neural activity. To resolve this issue, we placed a monopolar-stimulating electrode in the A11 region and made intracellular recordings from HVC projection neurons. Low intensity $(10 \mu \mathrm{A})$ electrical stimulation of A11 always (17 of 17 HVC cells) evoked short latency synaptic responses in HVC (Fig. 8D; median synaptic latency was $5.7 \pm 0.05$ ms; $n=17$ cells). Thus, the capacity of A11 neurons to follow LMAN activity and drive HVC activity at short latencies could enable this midbrain cell group to facilitate the rapid propagation of activity from LMAN to HVC.

To further test the idea that LMAN drives HVC activity through A11, we induced bursting activity in LMAN with BMI and inactivated A11 with GABA or muscimol while making extracellular recordings in LMAN, A11, and HVC. Under these conditions, HVC bursting activity continued to follow the onset of bursting activity in LMAN ( $n=2$ birds; data not shown). To explore whether A11 acts in parallel with one or more of the forebrain afferents of $\mathrm{HVC}$ to enable propagation of activity from LMAN to HVC, we also performed similar experiments where we sequentially inactivated NIf and A11. Following NIf inactivation, A11 inactivation was followed $5-15$ s later by a large burst of activity in HVC, after which LMAN bursting activity failed to drive MUA in HVC (Fig. $9 A, B$, insets; $n=3$ sites in 3 birds). These effects were most pronounced when the inactivation pipette was positioned within the $\mathrm{TH}^{+}$-region of $\mathrm{All}$, and were not observed when the pipette was positioned more lat- 
A

A11 ${ }_{\mathrm{HVC}}, \mathrm{TH}$ positive projecting neurons
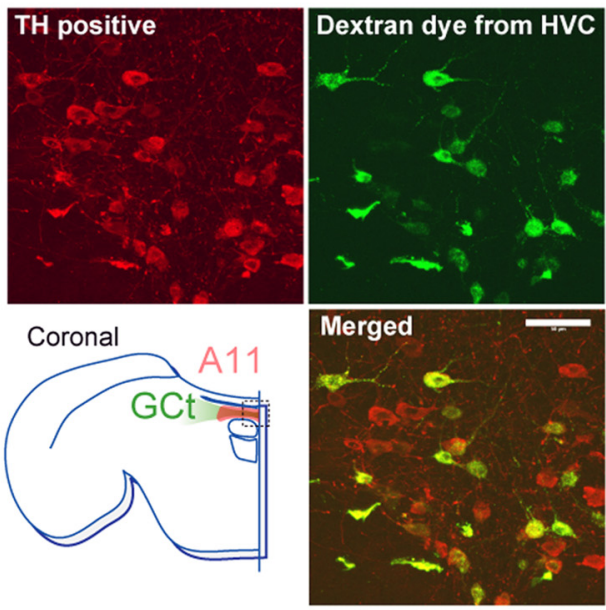

Sagittal

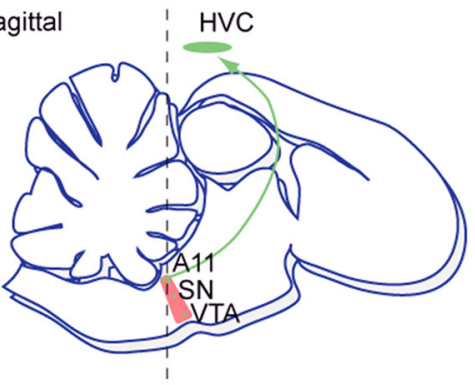

C

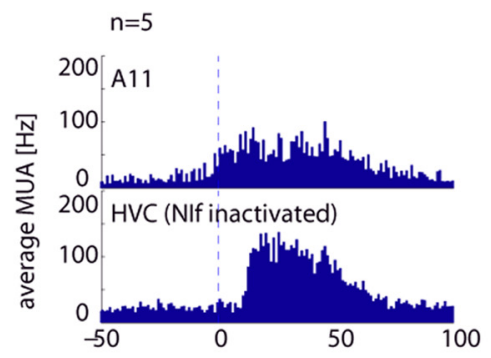

Relative time to LMAN burst onset [ms]
B

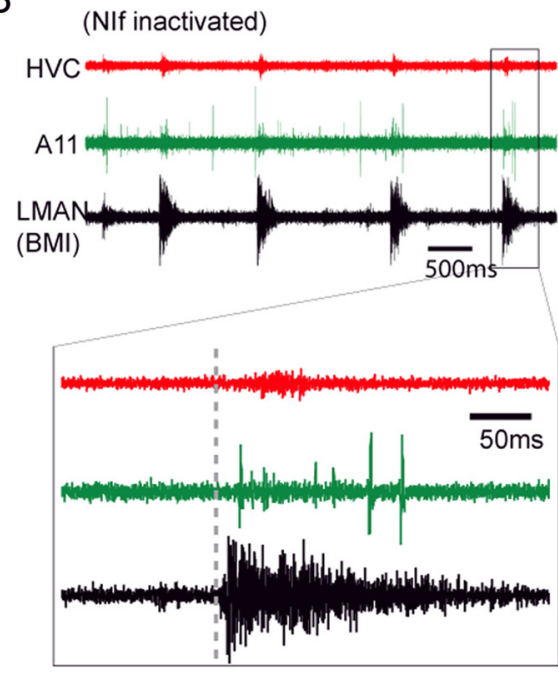

burst onset
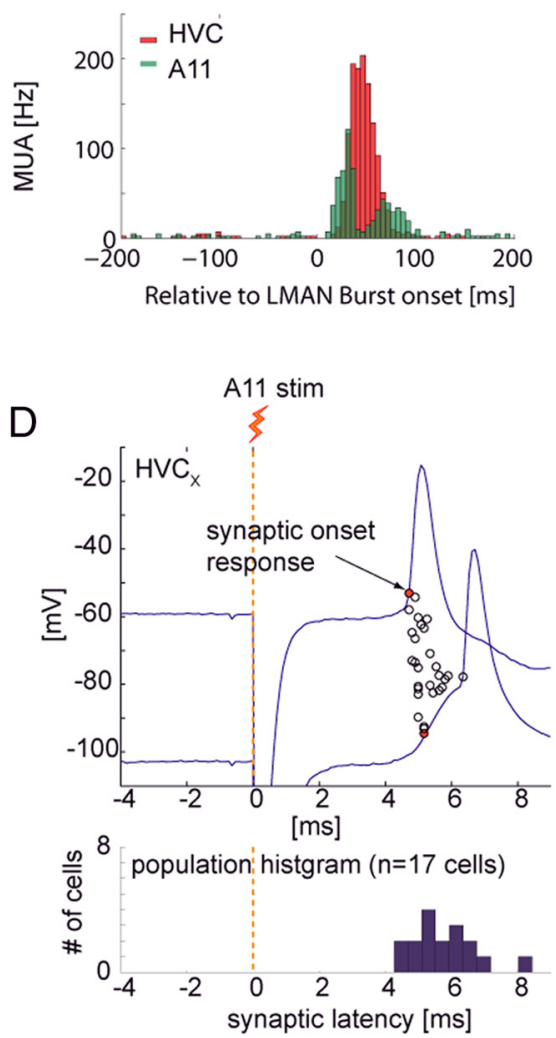

Figure 8. The dorsal midbrain dopaminergic area A11 displays properties consistent with a role in conveying activity from LMAN to HVC. $A$, Confocal images of TH-positive neurons in A11 (red; top left). HVC-projecting A11 ( $\mathrm{A} 11_{\text {HVC }}$ ) neurons ( green; top right) were labeled by injecting Dextran Alexa Fluor 488 tracer into HVC. Most of the A11 $1_{\text {HVC }}$ neurons near the midline $(0-700 \mu \mathrm{m})$ are TH-positive (merged; bottom right); scale bar is $50 \mu \mathrm{m}$ and applies to all confocal images. Bottom left, A drawing of a transverse section showing A11 ${ }_{\mathrm{HVC}}$ (red) and a more laterally-positioned population of TH-negative HVC-projecting neurons distributed over the central gray (GCt; green). B, MUA traces of HVC, A11, and LMAN recorded while bursting activity was induced in LMAN by BMI and NIf was inactivated with muscimol. LMAN-burst triggered average (bottom) of the firing rate histogram shows that activity in A11 follows the burst onset in LMAN earlier than the activity in HVC. C, Population average of LMAN burst-triggered PSTH of MUA recorded in A11 and HVC $[n=5$ triple recordings in 5 birds; A11: 5 out of 5 significant $(p<0.0006)$; HVC: 4 out of 5 significant ( $p<$ 1. $\left.\times 10^{-021}\right)$ ]. D, Top, Short latency excitatory synaptic responses evoked in an $\mathrm{HVC}_{x}$ neuron by electrical stimulation of $\mathrm{A} 11$ (10 $\mu \mathrm{A}$; dashed red line). Synaptic response onsets for multiple trials recorded from this cell are plotted with circles. Bottom, Distribution of the synaptic latency ( $n=17$ cells, $5.7 \pm 0.05 \mathrm{~ms}$ ).

erally in the population of $\mathrm{TH}^{-}$HVC-projecting neurons (Fig. 9C). These results support the idea that A11 and NIf can independently drive HVC, and that blocking activity in both of these structures is sufficient to prevent information flow from LMAN to HVC.
Augmenting LMAN activity increases sequence variability in adult zebra finches

Several lines of evidence suggest that LMAN interacts with HVC to modulate variability of syllable sequences. First, song motor control is hierarchically organized, with HVC influencing tem- 
poral aspects of song structure, including the sequencing and termination of song syllables (Vu et al., 1994; Yu and Margoliash, 1996; Hahnloser et al., 2002; Long and Fee, 2008). Second, electrical stimulation in LMAN and the ipsilateral HVC can trigger song truncation and restarts, and these effects are restricted to the same time windows in the song, a phenomenon that suggests an ipsilateral influence of LMAN on HVC that could be mediated by the circuitry we have described here (Wang et al., 2008). Third, inactivation of LMAN in juvenile zebra finches, which sing more variable syllable sequences, immediately reduces sequence variability (Olveczky et al., 2005). Finally, both sequence variability and singing-related bursting activity of LMAN neurons decline over the course of song development (Kao and Brainard, 2006), raising the possibility that sequence variability may be reinstated in older animals by reactivating LMAN.

To test this latter idea, we implanted custom microdialysis probes bilaterally in LMAN of adult [ $>90$ (PHD)] zebra finches and on alternating days infused either BMI $(10 \mathrm{~mm})$ or saline $(n=4$ hearing intact, $n=2$ deafened birds). To quantify effects on sequence variability, we used two measures, sequence consistency, which is a measure of how consistently the dominant syllable sequence transitions were generated (Scharff and Nottebohm, 1991), and song continuity, which is a measure of song duration that can capture changes in the bird's tendency to truncate its song (see Materials and Methods). Data were analyzed from song recordings made within $6 \mathrm{~h}$ after drug infusion, and syllables were identified semi-manually by using a support vector machine algorithm, allowing analysis of $\sim 10,000$ syllable transitions per day (see Materials and Methods).

Infusing BMI in the LMAN of adult zebra finches dramatically and reversibly augmented sequence variability (Fig. $10 A-D)$. Sequence consistency decreased on BMI days (Fig. $10 D$, circles; $n=4$ of 4 birds showed significant decreases, Man$\mathrm{n}$-Whitney $U$ test, $p<0.01$ ), an effect that was attributable to an increased generation of previously rare or less common syllable sequences. Furthermore, song continuity significantly decreased with BMI treatment in all birds (Fig. $10 \mathrm{E}$, circles; $n=4$ of 4 birds, Mann-Whitney $U$ test, $p<0.01$ ), an effect largely attributable to increased frequency of song truncation. In addition to these effects on global song features, we also noted that some syllables became noisier with BMI treatment (Fig. $10 F-H$ ). Because distorted auditory feedback can influence syllable transitions in some songbird species (Sakata and Brainard, 2006), and the noisier syllables associated with BMI treatexperiment. ${ }^{*} p<0.05$.
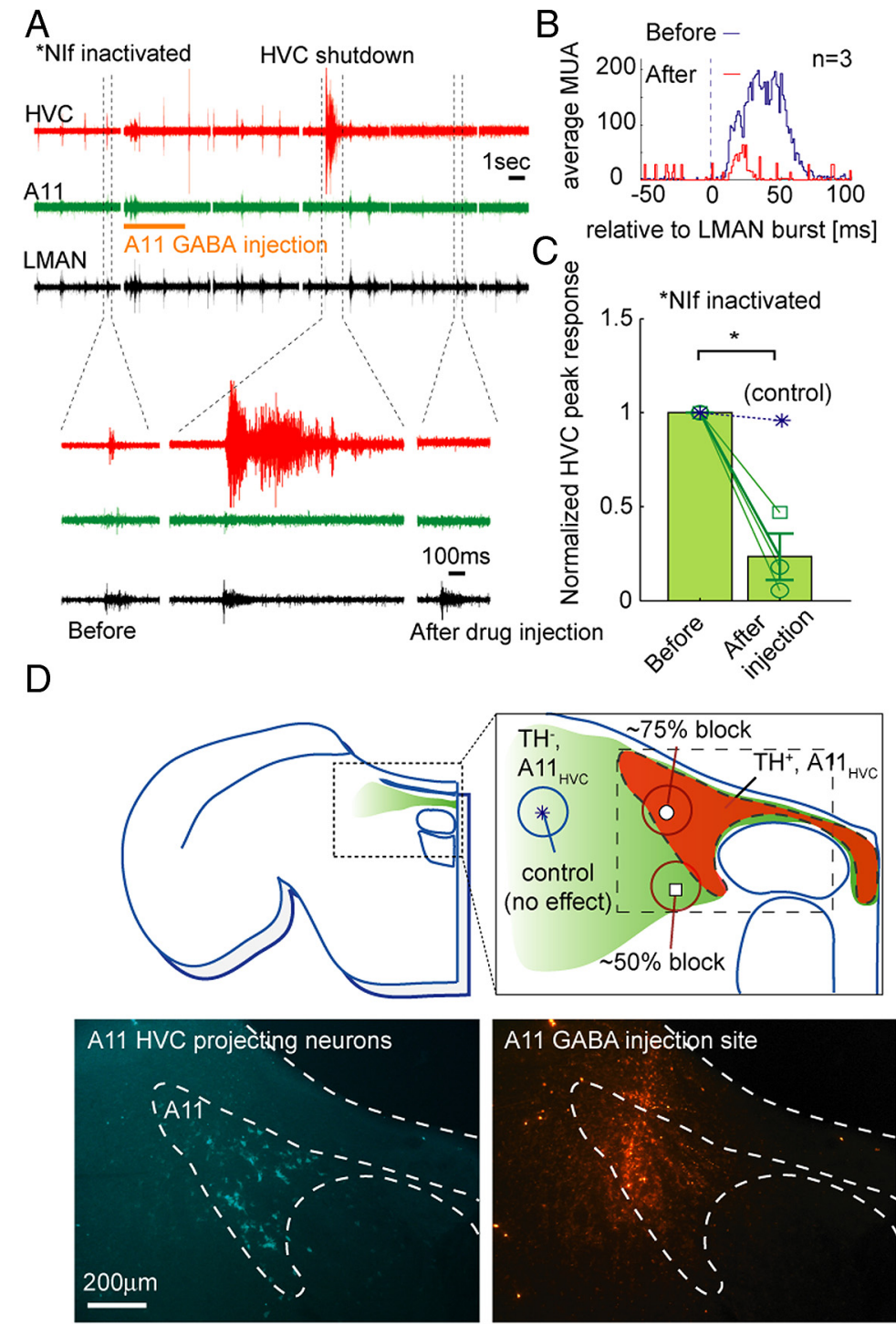

Figure 9. Inactivation of both A11 and Nlf blocks propagation of activity from LMAN to HVC. $A$, After inactivating NIf with muscimol ( $5 \mathrm{mM}$ ), BMI-induced LMAN bursting activity still drives HVC responses (left). Inactivating A11 with GABA ( $250 \mathrm{~mm}$, $500 \mathrm{nl}$ during orange bar) induced HVC shutdown $\sim 15 \mathrm{~s}$ after the onset of GABA injection (middle). After HVC shutdown, no response was detected in HVC and A11, despite continued bursting activity in LMAN. B, Averaged HVC MUA aligned to LMAN burst onsets $(n=3)$. C, Normalized HVC MUA responses aligned to LMAN burst onset were significantly reduced after HVC shutdown $(n=3$, paired $t$ test, $p<0.012$ ). Injecting GABA near the center of the A11 population strongly attenuated HVC responses to LMAN bursting (circles: $\sim 75 \%$ reduction). A muscimol injection centered $\sim 300 \mu \mathrm{m}$ ventral to the center of the distribution of A11 cells reduced the HVC response by $50 \%$ (square). An injection of muscimol placed $\sim 1200 \mu \mathrm{m}$ lateral from midline did not evoke any state change in HVC (asterisk). D, Top, Schematic diagram of the drug/tracer ( $250 \mathrm{~mm}$ GABA or 5 mm muscimol mixed with Dextran Alexa Fluor 594) injection sites, based on post hoc visualization of tracer and retrograde labeling from HVC with Dextran Alexa Fluor 488. Distribution of HVC-projecting A11 and more lateral GCt neurons are shown in green. The medial region contains TH-positive HVC-projecting cells (A11, red), while TH-negative HVC-projecting cells are more laterally distributed (light green). Bottom, The GABA injection site (red) and retrograde-labeled cells from tracer-injection in HVC (green) recovered $5 \mathrm{~d}$ after the inactivation

ment are a potential source of distorted feedback, we also tested whether BMI treatment affected sequence variability through an auditory feedback-dependent mechanism. However, measures of sequence consistency and song continuity decreased even in deafened adults when BMI was infused into LMAN (Fig. 10D, E, red triangles; $n=2$ of 2 birds, Mann-Whitney $U$ test, $p<0.01$ ). Therefore, artificially augmenting LMAN activity in adult zebra finches increases sequence variability through mechanisms that do not require auditory feedback. Along with prior studies in juvenile birds showing that LMAN inactivation can reduce se- 
A

Saline injection condition in pur820m, day1: saline

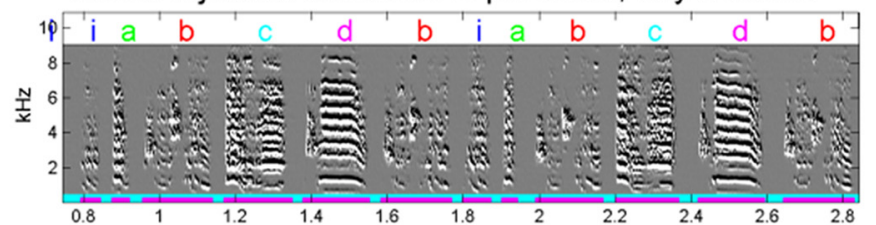

typical transition diagram

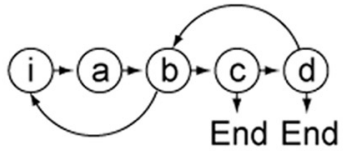

B Examples of variable syllable sequences in pur820m, day2: BMI transition "a-c"

transition "a-d"
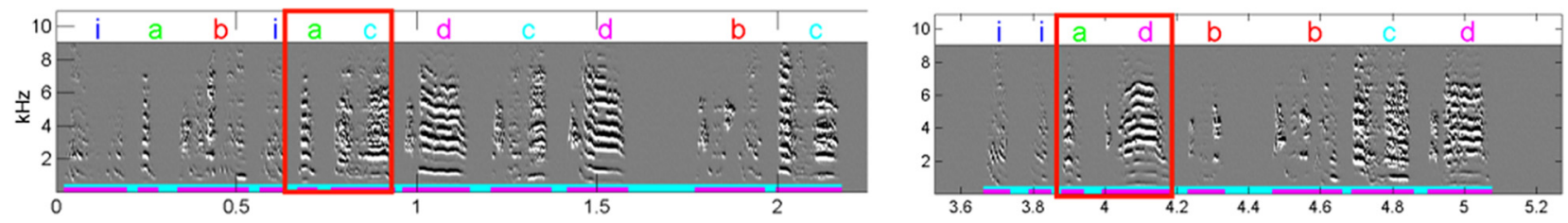

restart "d. d"

truncation "b-End"
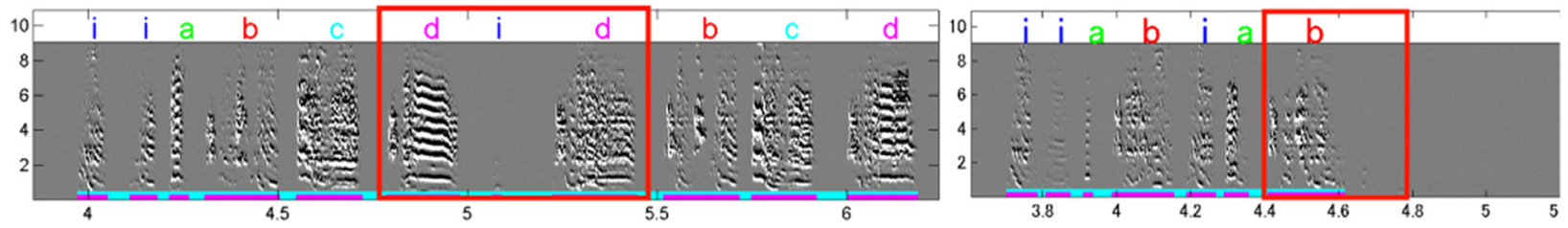

C

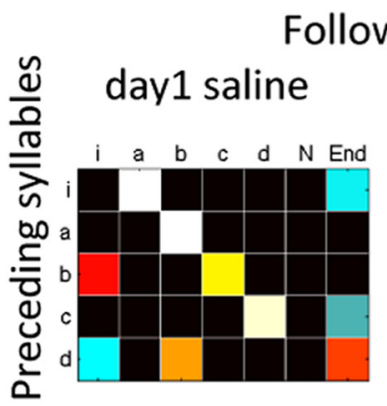

Following syllables

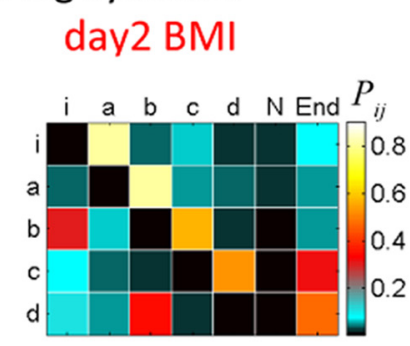

D

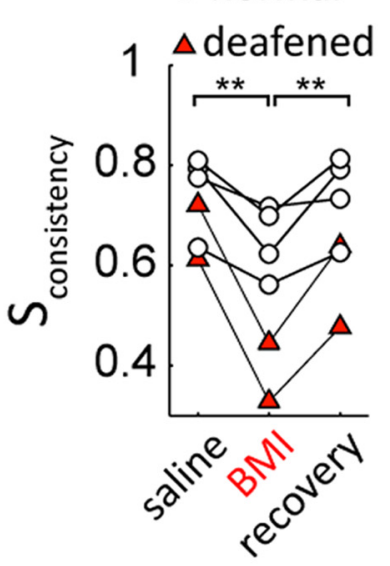

E

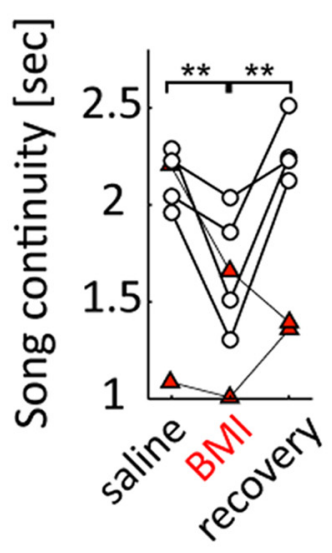

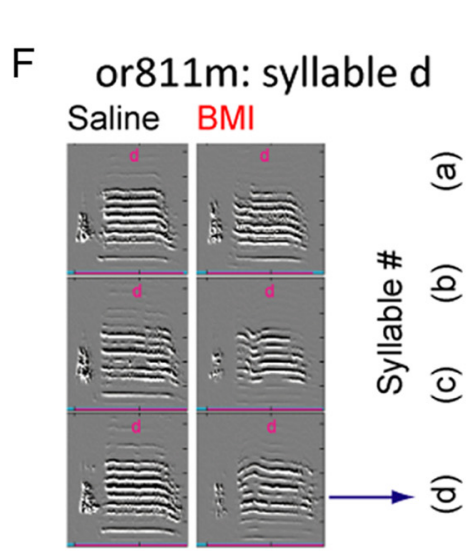

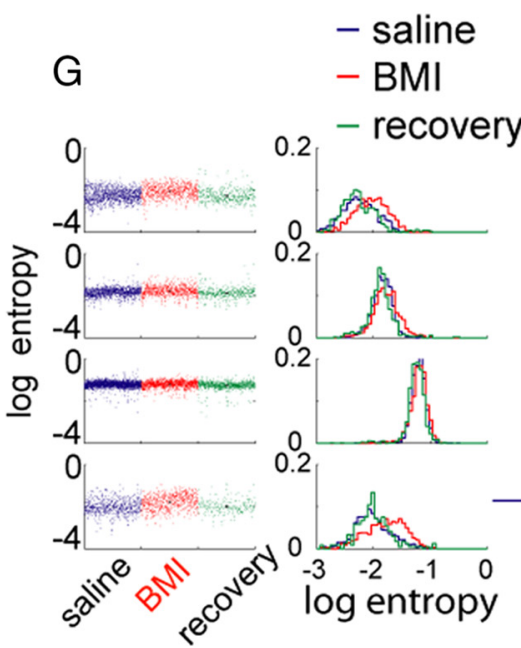

$\mathrm{H}$

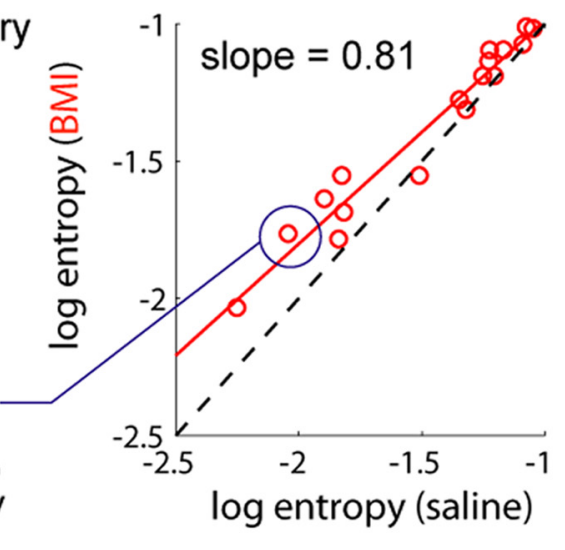

Figure 10. Augmenting LMAN activity with BMl infusion increases sequence variability and syllable entropy in adult zebra finches. A, Adult song recorded when saline was infused in LMAN, and typical transition diagram of song recorded under these conditions. $\boldsymbol{B}$, Examples of variable syllable sequences recorded when BMI (10 mM) was bilaterally infused in LMAN. (Figurelegend continues.) 
quence variability, the present findings support the idea that recurrent interactions from LMAN to HVC can modulate variability of vocal sequences.

\section{Augmenting LMAN activity in MMAN-lesioned birds is sufficient to induce sequence variability}

One potential challenge to interpreting the significance of the in vivo microdialysis experiments is the spatial extent of the spread of BMI into surrounding regions, which include $\mathrm{LMAN}_{\text {shell }}$, Area X, and MMAN, the latter of which is afferent to HVC. Although spread of BMI to $\mathrm{LMAN}_{\text {shell }}$ is unavoidable, and LMAN $\mathrm{L}_{\text {shell }}$ is speculated to influence sequence variability through a pathway that involves Ad and HVC (Bottjer and Altenau, 2010), the electrophysiological experiments we performed do not support such a circuit model (see Discussion for a more detailed consideration of this point). Furthermore, direct injection of BMI into Area X failed to induce bursting activity in Area X (K. Hamaguchi, unpublished observations), and thus is unlikely to account for the behavioral effects we observed. However, because the LMAN $_{\text {shell }}$-Ad pathway is theorized to affect sequence variability through MMAN (and HVC), and the microdialysis method may activate MMAN either directly (i.e., by diffusion) or indirectly (i.e., through the $\mathrm{LMAN}_{\text {shell }}$-Ad pathway), we conducted additional experiments to estimate drug diffusion from the probe into surrounding brain tissue and measure the behavioral effects of BMI infusion into LMAN in birds previously subjected to MMAN lesions.

To estimate drug diffusion, we infused fluorescent-dye tagged muscimol (1 mM, Muscimol BODIPY TMR-X conjugate; Invitrogen) into the probe at the end of the experiment. We chose muscimol because both $\mathrm{BMI}$ and muscimol are $\mathrm{GABA}_{\mathrm{A}}$ receptor binding molecules with similar molecular weights [607.5 (Muscimol BODIPY); 509.3 (BMI)]. After $9 \mathrm{~h}$ of drug infusion, we killed the animal and measured the fluorescence level in fixed brain sections (Fig. 11A,B). The fluorescence intensity (i.e., drug concentration) plotted as a function of distance from the probe was well fit with an exponential distribution. This outcome is consistent with the sta-

\section{$\leftarrow$}

(Figure legend continued.) C, Syllable transition matrix recorded on saline (left) and BMl (right) treatment days. $\boldsymbol{D}$, Sequence consistency scores of all normal hearing adults ( $n=4$ of 4 , circles) and deafened adults ( $n=2$ of 2, triangles) significantly decreased with BMl infusion in $\operatorname{LMAN}(p<0.01$, Mann-Whitney U test). E, Song continuity of adults decreased with BMI treatment in LMAN (salinevs BMI days, $p<0.01$, Mann-Whitney $U$ test, $n=4$ of 4 normal birds and $n=2$ of 2 deafened birds). $\boldsymbol{F}$, Examples of syllable level variability on control and BMI injection days. $\mathbf{G}$, Distribution of the log entropy (Wiener Entropy) of four syllables from bird or811m. $\boldsymbol{H}$, Syllable entropy significantly increased on BMI treatment days $(n=17$ syllables from three birds; linear regression; slope $=0.81$, $95 \%$ confidence limit $0.71-0.91)$. ${ }^{* *} p<0.01$.
B
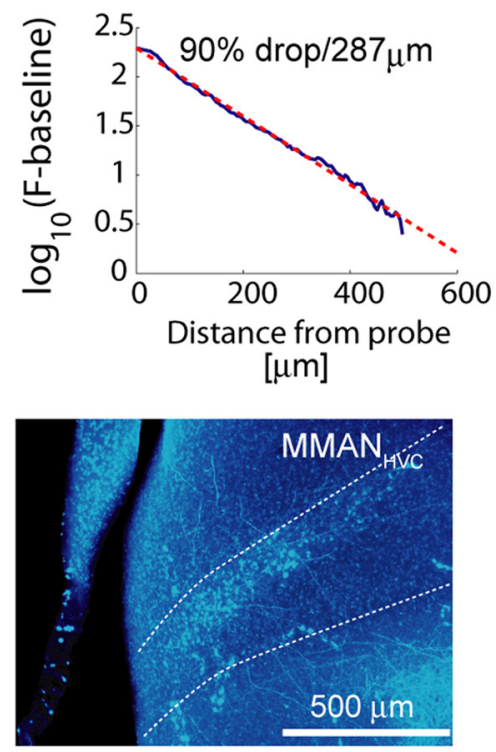

$\mathrm{E}$

* MMAN lesioned
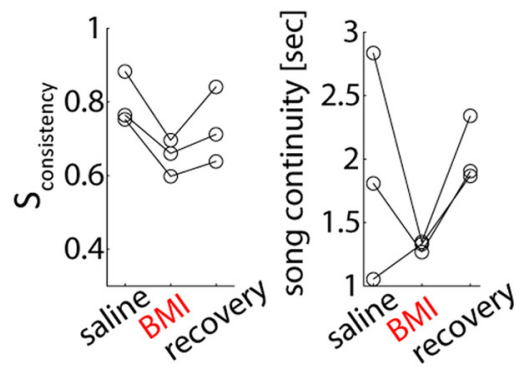

Figure 11. $\quad A$, Fluorescent photomicrograph of muscimol-BODIPY (1 mM) infused through dialysis probe visualized in a fixed tissue (he probe. C, Examples of an MMAN lesion visualized by Nissl-staining (left) and distribution of HVC

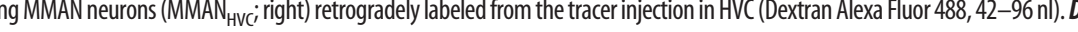

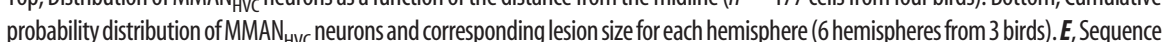
consistency score of MMAN-lesioned birds significantly decreased with BMI treatment in LMAN (saline vs BMI days, $p<0.01$, MannWhitney $U$ test, $n=3$ of 3 birds). Song continuity decreased with BMI treatment in LMAN in 2 of 3 birds (saline vs BMI day, $p<0.01$, Mann-Whitney U test, $n=2$ of 3 birds).

tionary distribution of a diffusion process in a one-dimensional axis with constant supply at one boundary (in this case, the dialysis probe). The spatial diffusion constant, obtained by fitting the exponential distribution was $0.00353\left[\log _{10} F / \mu \mathrm{m}\right]$, which translates to a $90 \%$ drop in concentration for every $\sim 280 \mu \mathrm{m}$. From this decay constant, and given the probe tip placement $1.85 \mathrm{~mm}$ from the midline, we estimated the drug concentration would be diluted 10,000fold $(\sim 100 \mathrm{nM}$, given the probe concentration of $10 \mathrm{mM})$ in the core of MMAN, which is $\sim 400 \mu \mathrm{m}$ from the midline. Therefore, we expect that the BMI concentration is too low to augment MMAN activity through direct pharmacological disinhibition.

Nonetheless, as we previously described (Fig. 5D-F), MMAN responds to BMI-induced bursting activity in LMAN, presumably though polysynaptic interactions between these two nuclei. Therefore, a remaining possibility is that augmented LMAN activity affects sequence variability through MMAN and not through other pathways involving NIf and A11. We therefore directly tested whether MMAN is involved in song sequence variability generated by augmented LMAN activity by making large 
Mammalian Cortico-BG circuit

Songbird Cortico-BG circuit

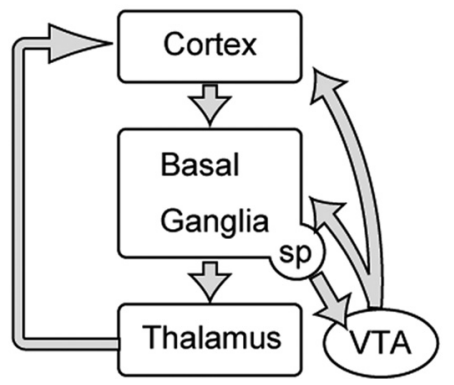

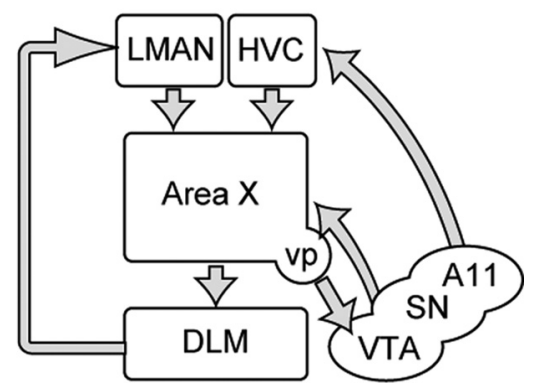

Figure 12. Similar structure of mammalian and songbird cortico-BG circuitry emphasizing mesocortical feedback. Midbrain dopaminergic cell groups in both mammals and birds provide recurrent input to the pallium: VTA neurons project to the prefrontal cortex and primary motor cortex in mammals, and A11 neurons project to HVC in songbirds. sp, Subpallidum; vp, ventral pallidum. bilateral lesions centered on MMAN (2\% ibotenic acid in PBS, 54 nl) and implanting dialysis probes bilaterally in LMAN $(n=3$ birds). Despite the almost complete absence of MMAN in these birds (Fig. 11C,D, see legend for quantification), we found that augmenting LMAN activity with BMI was sufficient to augment sequence variability, as reflected in decreased sequence consistency ( $n=3$ of 3, Mann-Whitney $U$ test, $p<0.01$ ) and song continuity scores $(n=2$ of 3 , Mann-Whitney $U$ test, $p<0.01$ ) (Fig. $11 E$ ). These additional control experiments indicate that infusing BMI into LMAN augments sequence variability through a pathway that does not involve MMAN, thus favoring a model where LMAN influences sequence variability through a circuit interaction that involves Area X, A11, NIf, and HVC (Fig. 12).

\section{Discussion}

This study strongly supports the idea that activity can propagate from LMAN to HVC to influence syllable sequence variability. Prior analyses detected correlations between HVC multiunit or interneuron activity and LMAN activity (Kimpo et al., 2003; Hahnloser et al., 2006), with both HVC-leading and LMANleading signatures. However, these earlier studies could not accurately estimate the precise temporal interactions between sparse-firing HVC PNs and LMAN neurons. The dual in vivo intracellular recordings used here revealed precisely correlated synaptic activity between HVC PN and LMAN cell pairs, and coherency analysis of their spontaneous membrane potential fluctuations revealed both HVC-leading and LMAN-leading signatures, consistent with these earlier studies. Moreover, electrically or chemically stimulating LMAN reliably evoked synaptic responses in HVC PNs, establishing the causality of the LMANleading peak in the coherency function, and underscoring that HVC and LMAN can interact bidirectionally. Notably, feedback from LMAN to HVC is rapid (i.e., $\sim 15 \mathrm{~ms}$ ) relative to both the duration of individual syllables $(\sim 100 \mathrm{~ms})$ and the syllable sequences constituting an entire song motif $(\sim 1 \mathrm{~s})$. Therefore, during singing, activity from LMAN could propagate to HVC over timescales sufficiently fast to influence syllable sequence variability, a view further supported by the current finding that augmenting LMAN activity could transiently increase sequence variability in adult birds.

A major issue is the nature of the circuitry that enables activity to flow from LMAN to HVC. The current findings show that in the anesthetized bird, activity propagates from LMAN to HVC through an ipsilateral pathway that involves Area X, the midbrain cell group A11, NIf, and possibly MMAN. One goal of future studies will be to identify the intermediate structures that link
Area X to A11 and NIf. Potential intermediates include the medial portion of dorsolateral thalamus (DLM) and the ventral pallidum (Vp), the latter of which innervates other midbrain dopaminergic cell groups, including the ventral tegmental area (VTA) and the substantia nigra (SN) (Gale et al., 2008). The electrophysiological experiments we performed also rule out a circuit model in which activity propagates from LMAN to HVC through RA and recurrent brainstem pathways involving Uva. Furthermore, inactivation of Ad failed to block propagation of activity from LMAN to HVC, arguing against the idea that chemical or electrical stimulation of LMAN inadvertently activated LMAN $_{\text {shell, }}$ which is speculated to communicate with HVC through Ad and intervening structures that include MMAN (Bottjer and Altenau, 2010). In fact, electrical stimulation in Ad or Av failed to evoke activity in HVC, raising doubts as to whether this putative circuit functions to mediate fast signaling between LMAN $_{\text {shell }}$ and HVC. Finally, MMAN lesions failed to prevent increases in syllable sequence variability induced by BMI perfusion into LMAN, further supporting a model in which LMAN modulates sequence variability through a pathway involving Area X, A11, and NIf.

The current study also sheds added light on midbrain-forebrain pathways in songbirds, the functions of which are not well understood but, by analogy to mammalian mesocortical pathways, may play an important role in motor learning (Hosp et al., 2011). Indeed, A11 neurons project to HVC and reliably follow LMAN bursting activity, providing suitable candidates for transmitting activity from LMAN to HVC. And although a subset of the A11 neurons that project to HVC are dopaminergic, electrical stimulation in A11 induced very short $(\sim 5 \mathrm{~ms})$ latency synaptic responses in $\mathrm{HVC}$, potentially through the co-release of glutamate, as observed in axon terminals of mammalian dopaminergic neurons (Kawano et al., 2006; Stuber et al., 2010). This capacity for fast signaling further qualifies A11 neurons as intermediaries that could rapidly relay activity from LMAN to HVC.

Nonetheless, the pathways and mechanisms that link LMAN to HVC display remarkable complexity and possible redundancy, because inactivating any single HVC afferent that responded to LMAN stimulation failed to block propagation of activity from LMAN to HVC. And although inactivating both A11 and NIf was sufficient to render HVC unresponsive to LMAN bursting activity, there was a noticeable delay between when A11 was inactivated and HVC stopped responding ( $5 \sim 15 \mathrm{~s})$, and even then HVC only stopped responding after a large and prolonged burst of activity. This delayed "shutdown" could reflect the time required to inactivate diffusely distributed A11 neurons and/or point to an unknown mechanism of the shutdown related to decreased dopaminergic tone in HVC. Although much remains to be clarified, the current findings suggest that LMAN activity influences sequence variability through striatal (Area X), dopaminergic (A11), and sensorimotor (NIf) structures.

Another goal of future studies will be to determine more precisely how these ipsilateral pathways function during singing. One clue that they could operate during singing is that high intensity unilateral stimulation in LMAN or the ipsilateral HVC in zebra finches can induce song truncations and restarts during similar limited periods in the motif (Wang et al., 2008). These 
findings are consistent with the idea that song is under the alternating control of the right and left hemispheres and that unilateral activation of LMAN can influence song only when the ipsilateral HVC is in control of singing (Wang et al., 2008; Long and Fee, 2008). These behavioral effects of ipsilateral LMAN and HVC stimulation parallel the lateralized LMAN influence on HVC activity observed here and further discount the possibility that LMAN influences HVC through RA and recurrent brainstem circuitry, which interacts bilaterally with HVC (Ashmore et al., 2008).

Previous studies have underscored the contribution of LMAN to syllable sequence variability in juvenile zebra finches undergoing sensorimotor learning [(Scharff and Nottebohm, 1991; Olveczky et al., 2005); for opposing evidence in the adult of another songbird species, see the study by Hampton et al. (2009)]. However, these loss of function experiments did not test whether augmenting LMAN activity was sufficient to reinstate high levels of sequence variability, and could not distinguish whether LMAN drives or gates sequence variability. The current finding that infusing BMI into the LMAN of adult zebra finches transiently increases both novel syllable transitions and song truncations supports the idea that LMAN actively drives sequence variability, while also providing confirmatory evidence that augmenting LMAN activity in adult birds is sufficient to drive syllable variability (Kao et al., 2005). However, because distorted auditory feedback can influence syllable transitions in some songbird species (Sakata and Brainard, 2006), it was unclear whether the elevated sequence variability is a direct effect of heightened LMAN activity or an indirect effect of elevated syllable variability, which could be detected as distorted feedback. Our finding that deafened birds infused with BMI in LMAN showed elevated sequence variability further supports the idea that heightened LMAN activity drives sequence variability through mechanisms that do not rely on auditory feedback.

The present study sheds light on how the output of BG pathway communicates with vocal motor regions, while adding significant complexity and spatiotemporal reach to an emerging paradigm that views the BG as a source of variability that fuels adaptive song learning (Andalman and Fee, 2009; Warren et al., 2011). Prior studies have shown that the songbird BG pathway plays a critical role in song learning and that LMAN can modulate the structure and variability of individual syllables through its connection to RA (Kao et al., 2005; Olveczky et al., 2005). This "point-wise" modulation is likely to play an important role in song learning (Doya and Sejnowski, 1995; Andalman and Fee, 2009; Warren et al., 2011). However, studies in machine learning indicate that complex motor sequences are learned more efficiently if modulation occurs at more than one level of the motor command hierarchy (Kaelbling et al., 1996; Barto and Mahadevan, 2003). Furthermore, the current findings raise the interesting possibility that heightened LMAN activity acts via A11 to rapidly elevate both sequence variability and dopamine levels in HVC, which could in turn promote synaptic plasticity important to learning. The $\mathrm{LMAN}_{\text {shell }}$ may also communicate with midbrain dopaminergic neurons through Ad (Gale and Perkel, 2008), and although Ad lesions in juvenile zebra finches have no immediate effects on song, these juveniles subsequently develop songs with abnormally high levels of sequence variability (Bottjer and Altenau, 2010). Therefore, the LMAN-A11 and the $\mathrm{LMAN}_{\text {shell }}-\mathrm{Ad}$ pathway may act in parallel during song learning to regulate sequence variability.

Numerous parallels exist between the songbird BG pathway and mammalian cortical basal ganglia circuits (Doupe et al.,
2005; Graybiel, 2005), and emerging evidence, including data presented here, indicates that the song system contains recurrent architecture similar to mammalian forebrain networks (Ashmore et al., 2008; Roberts et al., 2008). Although much remains to be determined about underlying mechanisms and circuitry, the ability of LMAN to influence syllable sequences and song terminations strengthens the functional parallel between songbird and mammalian BG pathways, the latter of which are implicated in the learning, initiation, and termination of motor sequences (DeLong, 1990; Westwater et al., 1998). The ability of BG pathways to efficiently modulate motor sequences could facilitate vocal learning, and may also account for the incorrect sequencing of articulatory gestures hypothesized to impair certain aspects of speech production following BG dysfunction (Pickett et al., 1998).

\section{References}

Andalman AS, Fee MS (2009) A basal ganglia-forebrain circuit in the songbird biases motor output to avoid vocal errors. Proc Natl Acad Sci U S A 106:12518-12523.

Ankri N, Legendre P, Faber DS, Korn H (1994) Automatic detection of spontaneous synaptic responses in central neurons. J Neurosci Methods 52:87-100.

Appeltants D, Absil P, Balthazart J, Ball GF (2000) Identification of the origin of catecholaminergic inputs to $\mathrm{HVc}$ in canaries by retrograde tract tracing combined with tyrosine hydroxylase immunocytochemistry. J Chem Neuroanat 18:117-133.

Ashmore RC, Renk JA, Schmidt MF (2008) Bottom-up activation of the vocal motor forebrain by the respiratory brainstem. J Neurosci 28:2613-2623.

Barto AG, Mahadevan S (2003) Recent advances in hierarchical reinforcement learning. Discrete Event Dyn S 13:341-379.

Bauer EE, Coleman MJ, Roberts TF, Roy A, Prather JF, Mooney R (2008) A synaptic basis for auditory-vocal integration in the songbird. J Neurosci 28:1509-1522.

Bottjer SW, Altenau B (2010) Parallel pathways for vocal learning in basal ganglia of songbirds. Nat Neurosci 13:153-155.

Bottjer SW, Miesner EA, Arnold AP (1984) Forebrain lesions disrupt development but not maintenance of song in passerine birds. Science 224:901-903.

Coleman MJ, Mooney R (2004) Synaptic transformations underlying highly selective auditory representations of learned birdsong. J Neurosci 24:7251-7265.

DeLong MR (1990) Primate models of movement disorders of basal ganglia origin. Trends Neurosci 13:281-285.

Doupe AJ, Perkel DJ, Reiner A, Stern EA (2005) Birdbrains could teach basal ganglia research a new song. Trends Neurosci 28:353-363.

Doya K, Sejnowski TJ (1995) A novel reinforcement model of birdsong vocalization learning. In: Advances in neural information processing systems (NIPS) (Tesauro G, Touretzky DS, Leen TK, eds.), pp 101-108. Cambride, MA: MIT.

Fee MS, Kozhevnikov AA, Hahnloser RH (2004) Neural mechanisms of vocal sequence generation in the songbird. Ann NY Acad Sci 1016:153-170.

Foster EF, Bottjer SW (2001) Lesions of a telencephalic nucleus in male zebra finches: Influences on vocal behavior in juveniles and adults. J Neurobiol 46:142-165.

Gale SD, Person AL, Perkel DJ (2008) A novel basal ganglia pathway forms a loop linking a vocal learning circuit with its dopaminergic input. J Comp Neurol 508:824-839.

Graybiel AM (2005) The basal ganglia: learning new tricks and loving it. Curr Opin Neurobiol 15:638-644.

Hahnloser RH, Kozhevnikov AA, Fee MS (2002) An ultra-sparse code underliesthe generation of neural sequences in a songbird. Nature 419:65-70.

Hahnloser RH, Kozhevnikov AA, Fee MS (2006) Sleep-related neural activity in a premotor and a basal-ganglia pathway of the songbird. J Neurophysiol 96:794-812.

Hampton CM, Sakata JT, Brainard MS (2009) An avian basal gangliaforebrain circuit contributes differentially to syllable versus sequence variability of adult bengalese finch song. J Neurophysiol 101:3235-3245. 
Hosp JA, Pekanovic A, Rioult-Pedotti MS, Luft AR (2011) Dopaminergic projections from midbrain to primary motor cortex mediate motor skill learning. J Neurosci 31:2481-2487.

Johnson F, Sablan MM, Bottjer SW (1995) Topographic organization of a forebrain pathway involved with vocal learning in zebra finches. J Comp Neurol 358:260-278.

Kaelbling LP, Littman ML, Moore AW (1996) Reinforcement learning: a survey. J Artif Intell Res 4:237-285.

Kao MH, Brainard MS (2006) Lesions of an avian basal ganglia circuit prevent context-dependent changes to song variability J Neurophysiol 96:1441-1455.

Kao MH, Doupe AJ, Brainard MS (2005) Contributions of an avian basal ganglia-forebrain circuit to real-time modulation of song. Nature 433:638-643.

Kawano M, Kawasaki A, Sakata-Haga H, Fukui Y, Kawano H, Nogami H, Hisano S (2006) Particular subpopulations of midbrain and hypothalamic dopamine neurons express vesicular glutamate transporter 2 in the rat brain. J Comp Neurol 498:581-592.

Kimpo RR, Theunissen FE, Doupe AJ (2003) Propagation of correlated activity through multiple stages of a neural circuit. J Neurosci 23:5750-5761.

Leblois A, Bodor AL, Person AL, Perkel DJ (2009) Millisecond timescale disinhibition mediates fast information transmission through an avian basal ganglia loop. J Neurosci 29:15420-15433.

Long MA, Fee MS (2008) Using temperature to analyse temporal dynamics in the songbird motor pathway. Nature 456:189-194.

Mooney R (2000) Different subthreshold mechanisms underlie song selectivity in identified HVc neurons of the zebra finch. J Neurosci 20:5420-5436.

Mooney R (2009) Neural mechanisms for learned birdsong. Learn Mem $16: 655-669$.

Mooney R, Prather JF (2005) The HVC microcircuit: the synaptic basis for interactions between song motor and vocal plasticity pathways. J Neurosci 25:1952-1964.

Olveczky BP, Andalman AS, Fee MS (2005) Vocal experimentation in the juvenile songbird requires a basal ganglia circuit. PLoS Biol 3:e153.

Pickett ER, Kuniholm E, Protopapas A, Friedman J, Lieberman P (1998) Selective speech motor, syntax and cognitive deficits associated with bilateral damage to the putamen and the head of the caudate nucleus: a case study. Neuropsychologia 36:173-188.

Rauske PL, Shea SD, Margoliash D (2003) State and neuronal classdependent reconfiguration in the avian song system. J Neurophysiol 89:1688-1701.
Roberts TF, Klein ME, Kubke MF, Wild JM, Mooney R (2008) Telencephalic neurons monosynaptically link brainstem and forebrain premotor networks necessary for song. J Neurosci 28:3479-3489.

Rosen MJ, Mooney R (2006) Synaptic interactions underlying songselectivity in the avian nucleus HVC revealed by dual intracellular recordings. J Neurophysiol 95:1158-1175.

Roy A, Mooney R (2007) Auditory plasticity in a basal ganglia-forebrain pathway during decrystallization of adult birdsong. J Neurosci 27:6374-6387.

Sakata JT, Brainard MS (2006) Real-time contributions of auditory feedback to avian vocal motor control. J Neurosci 26:9619-9628.

Scharff C, Nottebohm F (1991) A comparative study of the behavioral deficits following lesions of various parts of the zebra finch song system: implications for vocal learning. J Neurosci 11:2896-2913.

Stuber GD, Hnasko TS, Britt JP, Edwards RH, Bonci A (2010) Dopaminergic terminals in the nucleus accumbens but not the dorsal striatum corelease glutamate. J Neurosci 30:8229-8233.

Tchernichovski O, Mitra PP, Lints T, Nottebohm F (2001) Dynamics of the vocal imitation process: how a Zebra Finch learns Its song. Science 291:2564-2569

Toyama K, Kimura M, Tanaka K (1981) Cross-correlation analysis of interneuronal connectivity in cat visual cortex. J Neurophysiol 46:191-201.

Volgushev M, Chauvette S, Mukovski M, Timofeev I (2006) Precise longrange synchronization of activity and silence in neocortical neurons during slow-wave sleep. J Neurosci 26:5665-5672.

Vu ET, Mazurek ME, Kuo YC (1994) Identification of a forebrain motor programming network for the learned song of zebra finches. J Neurosci 14:6924-6934

Wang CZ, Herbst JA, Keller GB, Hahnloser RH (2008) Rapid interhemispheric switching during vocal production in a songbird. PLoS Biol 6:e250.

Warren TL, Tumer EC, Charlesworth JD, Brainard MS (2011) Mechanisms and time course of vocal learning and consolidation in the adult songbird. J Neurophysiol 106:1806-1821.

Westwater H, McDowall J, Siegert R, Mossman S, Abernethy D (1998) Implicit learning in parkinson's disease: evidence from a verbal version of the serial reaction time task. J Clin Exp Neuropsychol 20:413-418.

Wild JM (2004) Functional neuroanatomy of the sensorimotor control of singing. Ann N Y Acad Sci 1016:438-462.

Yu AC, Margoliash D (1996) Temporal hierarchical control of singing in birds. Science 273:1871-1875. 